\title{
Approximations for non-grey radiative transfer in numerical simulations of the solar photosphere
}

\author{
A. Vögler ${ }^{1}$, J. H. M. J. Bruls ${ }^{2}$, and M. Schüssler ${ }^{1}$ \\ 1 Max-Planck-Institut für Sonnensystemforschung ^, Max-Planck-Strasse 2, 37191 Katlenburg-Lindau, Germany \\ 2 Kiepenheuer-Institut für Sonnenphysik, Schöneckstrasse 6, 79104 Freiburg, Germany
}

Received 9 January 2004 / Accepted 24 March 2004

\begin{abstract}
Realistic simulations of solar (magneto-)convection require an accurate treatment of the non-grey character of the radiative energy transport. Owing to the large number of spectral lines in the solar atmosphere, statistical representations of the line opacities have to be used in order to keep the problem numerically tractable. We consider two statistical approaches, the opacity distribution function (ODF) concept and the multigroup (or opacity binning) method and provide a quantitative assessment of the errors that arise from the application of these methods in the context of $2 \mathrm{D} / 3 \mathrm{D}$ simulations. In a first step, the ODF- and multigroup methods are applied to a 1D model-atmosphere and the resulting radiative heating rates are compared. A number of 4-6 frequency bins is found to warrant a satisfactory modeling of the radiative energy exchange. Further tests in 2D model-atmospheres show the applicability of the multigroup method in realistic situations and underline the importance of a non-grey treatment. Furthermore, we address the question of an appropriate opacity average in multigroup calculations and discuss the significance of velocity gradients for the radiative heating rates.
\end{abstract}

Key words. Sun: photosphere - radiative transfer - methods: numerical

\section{Introduction}

The photosphere is the layer within the solar atmosphere where the energy transport changes from mainly convective to mainly radiative. Hence the radiation field plays a crucial role in the overall energy balance of the photosphere and requires a detailed modelling in numerical simulations aiming at a realistic description of the solar conditions.

Since the radiation pressure can be neglected in the solar atmosphere and since all material velocities are much smaller than the speed of light, in the framework of (magneto-) convection simulations the radiation field enters the set of $(\mathrm{M}) \mathrm{HD}$ equations via the radiative heating rate per unit volume

$Q_{\mathrm{rad}}=-\nabla \cdot \boldsymbol{F}=4 \pi \varrho \int \kappa_{v}\left(J_{v}-S_{v}\right) \mathrm{d} v$

Here $\boldsymbol{F}=\int \boldsymbol{F}_{\nu} \mathrm{d} v$ is the frequency-integrated radiative flux, $\varrho$ the mass density, and $\kappa_{v}$ the opacity. $J_{v}=\int I_{v}(\omega) \mathrm{d} \omega / 4 \pi$ is the (angle-averaged) monochromatic mean intensity, $I_{v}(\omega)$ is the direction-dependent intensity, and $S_{v}$ is the source

Send offprint requests to: A. Vögler,

e-mail: avoegler@linmpi.mpg.de

^ Formerly: Max-Planck-Institut für Aeronomie. function. In order to calculate the heating rate, the radiative transfer equation,

$\frac{\mathrm{d} I_{v}}{\mathrm{~d} s}=-\kappa_{v} \varrho\left(I_{v}-S_{v}\right)$

has to be solved for $I_{v}(\omega)$ at each gridpoint for all frequencies and directions. From this $\boldsymbol{F}_{v}$ (or $J_{v}$ ), and finally $Q_{\text {rad }}$ can be derived. Since the total opacity in the solar atmosphere comprises of the order of $10^{6}$ atomic and molecular spectral lines, a number of roughly $10^{6}-10^{7}$ frequency points is necessary in order to model the detailed frequency dependence. While this direct approach is feasible in calculating 1D static models, the computational cost is intolerable in time-dependent 2D or 3D simulations. The most radical simplification of the problem is achieved by the grey approximation, replacing the frequencydependent opacity by an averaged value, e.g. the Rosseland mean. The grey approach in combination with the diffusion approximation may be appropriate in the optically dense subphotospheric regions, where the radiation field is in local equilibrium with the gas, it is however unsatisfactory in the optically thinner regions where the radiation transfer becomes nonlocal. Here it is also necessary to explicitly account for line opacities, since line-blanketing effects have a considerable impact on both the photospheric energy balance and the emergent intensities. It is well known that the inclusion of line opacities in calculations of stellar model atmospheres strongly modifies the resulting teperature profiles, leading to considerably 
cooler outer layers, while the temperature is raised in deeper regions (the line cooling and backwarming effects, e.g., Mihalas 1978, p. 167). Therefore, a statistical treatment of line opacities which conserves the non-grey character of the radiation transport while drastically reducing the computational expense is highly desirable. Among the strategies devised to tackle this problem are the method of opacity sampling (Peytremann 1974; Sneden et al. 1976), the concept of Opacity Distribution Functions (ODFs, Strom \& Kurucz 1966; Mihalas 1967), and the multigroup (or opacity binning) method (Nordlund 1982; Ludwig 1992; Ludwig et al. 1994; Skartlien 2000). In the context of time-dependent three-dimensional simulations, for which the radiative transfer must be solved for every timestep, the only feasible approach - given the currently available computing resources - is the multigroup method, since it implies the most drastic reduction in computational cost and allows for a satisfactory approximation of the frequency-dependence with only a few representative frequency bins.

While the multigroup approach has been tested and sucessfully applied to calculations of 1D static model atmospheres (Ludwig 1992), the application in the context of timedependent 2D/3D simulations is accompanied by new sources of errors not encountered in the static 1D case like, for instance, strong lateral variations of the atmospheric properties. In order to gain confidence in the applicability of this approach in simulations, we performed a series of tests of the multigroup method for several 1D and 2D cases with the solution based on Opacity Distribution Functions serving as the reference solution.

In Sect. 2 the ODF concept and the multigroup method are briefly described. The test results are presented in Sect. 3. We always assume local thermodynamic equilibrium (LTE), implying $S_{v}=B_{v}$, thereby neglecting scattering effects. Line scattering becomes important in chromospheric heights where radiative cooling takes place in a few strong spectral lines, but these regions are currently beyond the scope of our project. The assumption of LTE also implies that the number densities of ions and the population numbers of excitation states are instantaneous functions of the local thermodynamical state of the system only. For this reason, the opacities can be obtained from pre-compiled tables without having to solve the system of rate equations for each gridpoint and timestep during a simulation run, which is an important requirement for the practicability of the aforementioned methods.

\section{Opacity distribution functions and the multigroup method}

\subsection{Opacity distribution functions}

The concept of opacity distribution functions (see e.g., Strom \& Kurucz 1966; Mihalas 1967) is based on the observation that physically relevant quantities of the radiation field like mean intensity $J=\int J_{v} \mathrm{~d} v$ and total heating rate $Q=4 \pi \varrho \int \kappa_{v}\left(J_{v}-\right.$ $\left.B_{v}\right) \mathrm{d} v$ are integrals over frequency and hence should not depend crucially on the detailed frequency structure of the opacity. The basic idea of the ODF approach is to divide the frequency spectrum into intervals $\Delta v_{i}$ (typically $10^{2}-10^{3}$ ) and replace the detailed spectrum of line-opacities within each interval by a smooth distribution function. In practice, the distribution functions are approximated by step functions with a small number of steps, and frequency-integrated quantities are calculated by summing over all ODF-intervals and steps, reducing the computational effort by a factor of the order $10^{3}$. The reordering of opacities within a given ODF-interval is based on the assumption that frequency- and height-dependence of the detailed opacity spectrum are decoupled. However, since changes in the states of excitation and ionisation of the gas, overlapping lines of two elements with different opacity variations with depth, or relative line shifts due to velocity gradients cause changes of the line spectrum with height within a given ODF-interval, this assumption does not hold strictly. While calculations of static model atmospheres (e.g., Gustafsson et al. 1975) show no significant error due to the ODF approximation, velocity gradients may introduce an additional error in time dependent MHD-simulations. We address this question briefly in Sect. 3.6.

\subsection{The multigroup method}

In this section we describe the multigroup approach proposed by Nordlund (1982) in the form developed by Ludwig (1992). The basic idea is to divide the frequency spectrum into a small number (typically 3-5) of (usually non-contiguous) subsets (called "bins"), $\Omega_{i}$, according to the strength of the corresponding opacities. This way, continuum opacities and lines of different strength are pooled in seperate bins and represented by bin-averaged opacities. Introducing the bin-integrated quantities $I_{i}=\int_{\Omega_{i}} I_{\nu} \mathrm{d} v$ and $B_{i}=\int_{\Omega_{i}} B_{\nu} \mathrm{d} v$, and assuming that the classification into bins remains fixed for all points along a ray, the radiative transfer equation can be written for each bin in the form

$$
\begin{aligned}
\frac{\mathrm{d} I_{i}}{\mathrm{~d} s} & =-\int_{\Omega_{i}} \kappa_{v} \varrho\left(I_{v}-B_{v}\right) \mathrm{d} v \\
& \simeq-\bar{\kappa}_{i} \varrho\left(I_{i}-B_{i}\right) .
\end{aligned}
$$

The introduction of a bin-averaged opacity $\bar{\kappa}_{i}$ in Eq. (3) is the essential step in the multigroup approach, because here a large part of the frequency integration has been done before the integration along the ray is carried out. For a given ray Eq. (3) is solved once for each bin, thereby drastically reducing the computational effort. This procedure is strictly correct only if $\kappa_{v}$ is constant over the whole bin, so one makes the implicit assumption that the depth-dependence of $\kappa_{v}$ (and thus the $\tau_{v}$ scales) are identical or at least very similar for the frequencies within one bin. Since in general this assumption holds only approximately, it is necessary to define a bin-averaged opacity. In optically thick regions the angle-averaged intensity $J_{v}$ approaches $B_{v}$ and the bin-integrated heating rate $Q_{\mathrm{rad}, i}=\int_{\Omega_{i}} \kappa_{\nu} \varrho\left(J_{v}-B_{v}\right) \mathrm{d} v$ tends to zero, so the definition of a mean opacity is not critical in this regime. The obvious choice is the Rosseland mean, as radiative transfer is effectively local and can be described as a diffusive process for $\tau_{v} \gg 1$ :

$\boldsymbol{F}_{v}=-\frac{4 \pi}{3 \kappa_{v}} \frac{\mathrm{d} B_{v}}{\mathrm{~d} T} \nabla T$ 
For the bin-integrated radiative flux one has

$$
\boldsymbol{F}_{i}=-\frac{4 \pi}{3 \bar{\kappa}_{R, i}} \int_{\Omega_{i}} \frac{\mathrm{d} B_{v}}{\mathrm{~d} T} \mathrm{~d} v \nabla T
$$

with the Rosseland mean defined by

$$
\frac{1}{\bar{\kappa}_{R, i}}=\frac{\int_{\Omega_{i}} \frac{1}{\kappa_{v}} \frac{\mathrm{d} B_{v}}{\mathrm{~d} T} \mathrm{~d} v}{\int_{\Omega_{i}} \frac{\mathrm{d} B_{v}}{\mathrm{~d} T} \mathrm{~d} v} .
$$

This definition gives the correct bin-integrated radiative fluxes.

In the regime $\tau_{v} \lesssim 1$, the diffusion approximation breaks down and the Rosseland mean is no longer applicable. Being a harmonic mean, it tends to underestimate the line opacities. For optically thin regions an averaging presciption for the opacity can be obtained by requiring that the correct energy balance within each bin is maintained:

$$
\int_{\Omega_{i}} \kappa_{v}\left(J_{v}-B_{v}\right) \mathrm{d} v=\bar{\kappa} \int_{\Omega_{i}}\left(\bar{J}_{v}-B_{v}\right) \mathrm{d} v .
$$

For a given choice of $\bar{\kappa}, \bar{J}_{v}$ is the angle-averaged intensity that results from replacing $\kappa_{v}$ with $\bar{\kappa}$ in the radiative transfer equation and solving for $I_{v}$. (The source function $B_{v}$ is still frequency-dependent, hence the index $v$ in $\bar{J}_{v}$ ).

If one further assumes that

$$
\int_{\Omega_{i}} \bar{J}_{v} \mathrm{~d} v \simeq \int_{\Omega_{i}} J_{v} \mathrm{~d} v
$$

then Eq. (7) is equivalent to

$$
\int_{\Omega_{i}}\left(\kappa_{v}-\bar{\kappa}\right)\left(J_{v}-B_{v}\right) \mathrm{d} v=0 .
$$

$J_{v}$ is not known a priori, but for $\tau_{v} \ll 1$ it can be approximated by developing $B_{v}\left(\tau_{v}\right)$ in a Taylor series and applying the $\Lambda_{v}$-operator that connects $B_{v}$ and $J_{v}$ (see e.g., Mihalas 1978, p. 59 for details). The result is

$$
J_{v}-B_{v} \simeq-\frac{1}{2} B_{v}
$$

By inserting Eq. (10) into Eq. (9) and solving for $\bar{\kappa}$, the Planck mean,

$\bar{\kappa}_{P, i}=\frac{\int_{\Omega_{i}} \kappa_{v} B_{\nu} \mathrm{d} v}{\int_{\Omega_{i}} B_{\nu} \mathrm{d} v}$,

is obtained. This "derivation" is far from rigorous and should be viewed as an indication that the Planck mean can be expected to yield a reasonable approximation of $Q_{\text {rad }}$ for $\tau_{v} \ll 1$.

Ludwig (1992) uses a hybrid scheme that models a steep transition from $\bar{\kappa}_{P}$ to $\bar{\kappa}_{R}$ at a threshold optical depth of $\tau_{0}=0.35$ :

$\bar{\kappa}_{i}=2^{-\frac{\tau_{i}}{\tau_{0}}} \bar{\kappa}_{P, i}+\left(1-2^{-\frac{\tau_{i}}{\tau_{0}}}\right) \bar{\kappa}_{R, i}$.

The index $i$ indicates that the means $\bar{\kappa}_{P, i}$ and $\bar{\kappa}_{R, i}$ are to be evaluated for each frequency bin, $\Omega_{i}$. For each bin, $\bar{\kappa}_{i}$ can be tabulated on a $(p, T)$ grid for subsequent use in simulations. Since the optical depth $\tau_{i}$ associated with local values of $p$ and $T$ is not known prior to a simulation run, it has to be estimated.
Assuming that the gas is hydrostatically stratified and that the depth-dependence of $\bar{\kappa}_{R, i} \varrho$ is dominated by an exponential increase of $\varrho$ with geometrical depth, Ludwig (1992) derives the approximate expression

$\tau_{i} \simeq \frac{\bar{\kappa}_{R, i} p}{g_{\odot}}$

where $g_{\odot}$ is the gravitational acceleration in the photosphere.

A key step in the multigroup method is the choice of an appropriate criterion for sorting frequencies into bins. The rationale for any sorting rule must be to group together opacities that are of the same order of magnitude and show similar behaviour as a function of depth. A simple choice is the so called $\kappa$-sorting, starting from the choice of a fixed height $z_{0}$ (or, alternatively, a representative pair of values $p_{0}$ and $T_{0}$ ) in a reference atmosphere, the frequency bins are then constructed according to the spectral shape of $\kappa_{v}\left(z_{0}\right)$. A number of threshold values $\kappa^{l}$ (the index $l$ labeling the bin, not to be mistaken as an exponent) which divide the range of opacity values into intervals, are selected. A frequency $v$ becomes part of a given bin if $\kappa_{v}\left(z_{0}\right)$ lies within the corresponding interval:

$v \in \Omega_{l} \quad$ if $\quad \kappa^{l} \leq \kappa_{v}\left(z_{0}\right)<\kappa^{l+1}$.

A reasonable choice for $z_{0}$ is the height where the continuum at $500 \mathrm{~nm}$ forms, $\tau_{500}\left(z_{0}\right) \approx 1$. Here a large part of the emerging flux originates and the energy exchange between gas and radiation field plays an important role, requiring an accurate modelling of the opacities. The threshold values $\kappa^{l}$ have to cover several orders of magnitude in order to account for the line opacities that become important at greater heights.

One disadvantage of the $\kappa$-sorting procedure is that it does not provide a reasonable selection criterion for frequencies that are optically thick at $z_{0}$, since the opacity values at $z_{0}$ have no impact on the radiative energy exchange taking place at such frequencies, as the main contribution for a given frequency $v$ to the heat exchange $Q_{v}$ comes from a height around $\tau_{v} \approx 1$. It seems therefore advantageous to sort the frequencies according to the opacity in this region instead of using a fixed reference height. This $\tau$-sorting, suggested by Ludwig (1992) applies the following strategy. First a 1D reference atmosphere is chosen. Then $\kappa_{v} \varrho$ is integrated along $z$ for all frequencies and the geometrical height corresponding to $\tau_{v}=1$ is determined. Measuring height in terms of a reference optical depth scale $\tau_{\text {ref }}$ (e.g. the Rosseland optical depth), threshold heights $\tau_{\text {ref }}^{l}$ are chosen. Then $v$ belongs to bin $\Omega_{l}$, if $\tau_{v}=1$ is reached within the corresponding interval of Rosseland optical depth:

$v \in \Omega_{l} \quad$ if $\quad \tau_{\text {ref }}^{l} \geq \tau_{\text {ref }}\left(\tau_{v}=1\right)>\tau_{\text {ref }}^{l+1}$.

If one assumes that the $\tau_{v}$ scales are mainly governed an exponential depth-dependence of $\varrho$, then an approximate relation similar to Eq. (13) holds, implying that $\tau$-sorting, (like $\kappa$-sorting), groups together opacities of the same magnitude. Furthermore, in contrast to $\kappa$-sorting, in the $\tau$-sorting method all opacity values between $\tau_{v}=0$ and $\tau_{v}=1$ contribute to the selection process and the frequencies of one bin have the same optical depth for at least one height $\tau_{\text {ref }}\left(\tau_{v}=1\right)$. Consequently, one may expect that in the region $0<\tau_{v} \leq 1$ where a nongrey description of radiation transfer is important the $\tau_{v}$-scales 


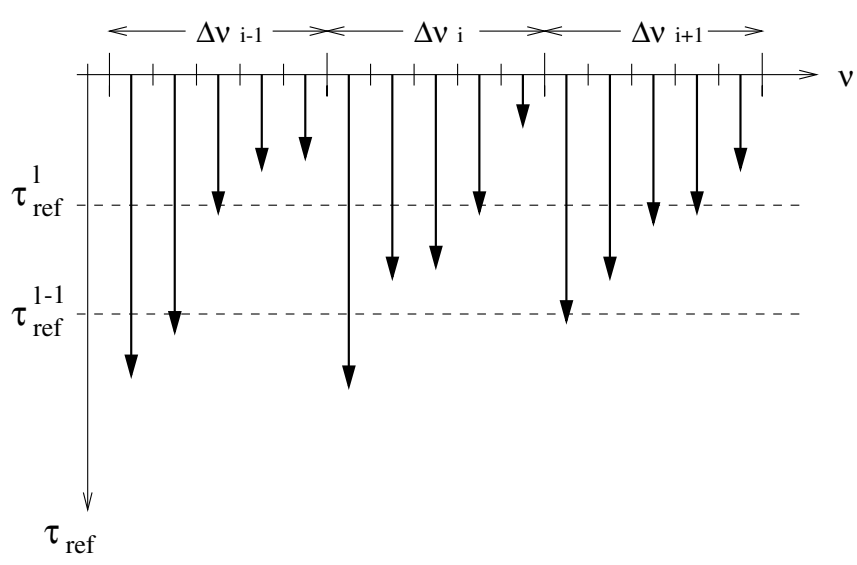

Fig. 1. Schematic illustration of the $\tau$-sorting procedure using ODFs. Within each ODF interval $\Delta v_{i}$, the ODF steps $\Delta v_{i j}$ are sorted to frequency bins $\Omega_{l}$, depending on the height where $\tau_{i j}=1$ is reached (indicated by bold arrows).

within one bin are similar, which is the major requirement for the validity of the multigroup approach. For greater depths, the way frequencies are gathered may be inappropriate, but this shortcoming is less severe as radiation transfer becomes increasingly grey in these regions anyway.

The $\tau$-sorting procedure can be realized in a convenient way by using ODFs. For a given step $j$ within ODF interval $\Delta v_{i} \kappa_{i j} \varrho$ is integrated along $z$, which gives $\tau_{i j}$ as a function of height in the reference atmosphere (see Fig. 1). The ODF steps are then sorted into bins $\Omega_{l}$. Bin-integrated quantities are obtained as weighted sums over the ODF steps in a given bin, viz.

$B_{l}=\sum_{i} \Delta v_{i} B_{\Delta v_{i}} \sum_{j(i, l)} w_{j(i, l)}$

and

$\bar{\kappa}_{P, l}=\frac{1}{B_{l}} \sum_{i} \Delta v_{i} B_{\Delta v_{i}} \sum_{j(i, l)} w_{j(i, l)} \kappa_{i j(i, l)}$,

where, for a given bin index $l$, the index $j(i, l)$ runs over those steps in $\Delta v_{i}$, which are elements of $\Omega_{l} . B_{\Delta v_{i}}$ denotes the average of $B$ over the ODF interval $\Delta v_{i}$. The effect of the sorting procedure on the opacity spectrum is illustrated in Figs. 2 and 3. Figure 2 shows the ODF-based opacity from the ATLAS9 stellar atmosphere code (Kurucz 1993) for $T=4470 \mathrm{~K}$ and $\log p=$ 4.12 (corresponding to a continuum optical depth at $500 \mathrm{~nm}$ $\log \tau_{500}=-2$ in the solar atmosphere). After applying the tausorting procedure with five opacity bins the spectrum is a step function which can assume five discrete values (Fig. 3).

In contrast to the ODFs, which approach the exact opacity spectrum in the limit of infinitely small ODF intervals, the multigroup solution can not be expected to converge to the exact solution (i.e. the solution based on the detailed reference spectrum which was used in the sorting process) in the limit of a very large number of opacity bins. This is due to the fact that, even for infinitely small intervals between bin-levels $\tau_{\text {ref }}^{l}$ (or $\kappa^{l}$ in the case of $\kappa$-sorting), each bin will comprise frequencies from different parts of the spectrum with different height profiles of the corresponding opacities. It should be noted that

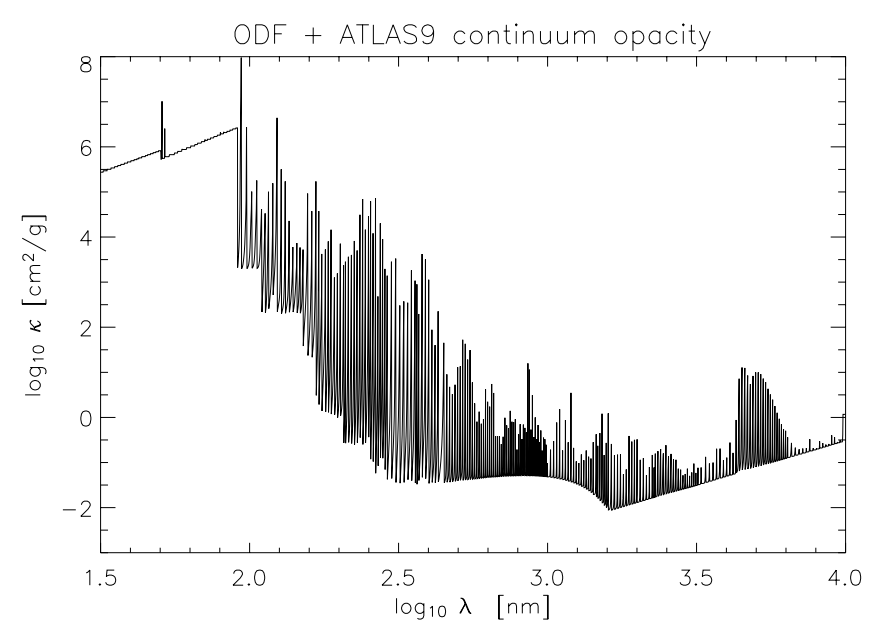

Fig. 2. Monochromatic opacity as a function of wavelength for $T=$ $4470 \mathrm{~K}$ and $\log p=4.12$, corresponding to $\log \tau_{500}=-2$ in the solar atmosphere. ATLAS9 (Kurucz 1993) Opacity Distribution Functions are used to model the line opacities.

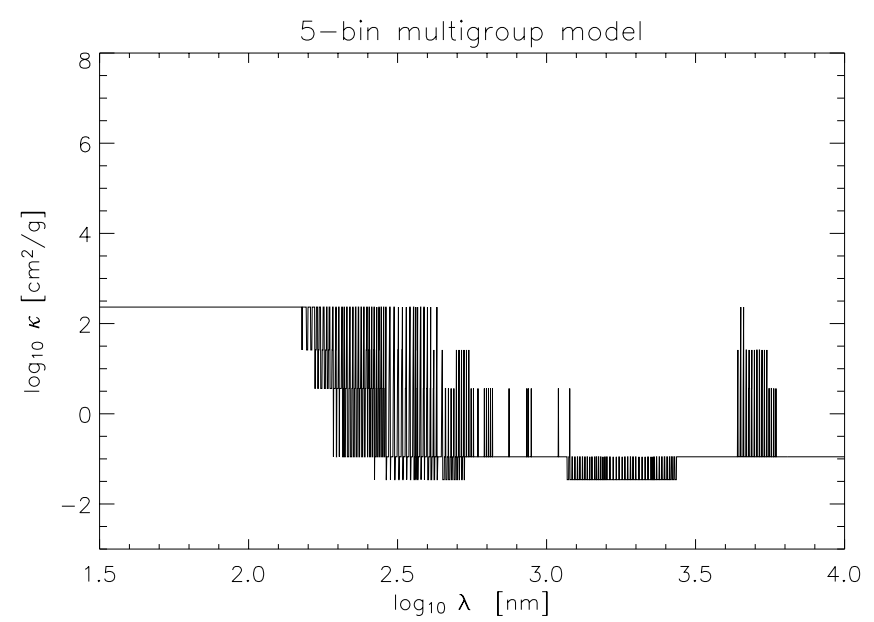

Fig. 3. Opacity as a function of wavelength for a $\tau$-sorted multigroup model with five opacity bins, for the same thermodynamical conditions as in Fig. 2.

using ODFs as basis for sorting frequencies introduces an additional error since the rearrangement of spectral lines inherent in the ODF concept affects the way frequencies are classified. However, since the ODF solution approximates the exact solution well, the error incurred this way is small compared to the consequence of the binning procedure (see Sect. 3.5).

The choice of a reference atmosphere is another possible source of error connected with the $\tau$-sorting scheme. It is plausible that the opacity binning method with $\tau$-sorting shows good results in calculations of static 1D model atmospheres if the "exact" solution (i.e., the 1D atmosphere resulting from the ODF approach) is chosen as the reference atmosphere. In multidimensional time-dependent simulations, however, the physical parameters may deviate considerably from a $1 \mathrm{D}$ reference stratification. As a consequence, the assignment of frequencies to bins might lead to an inappropriate representation of the opacities. In order to test the applicability of the $\tau$-sorting procedure in non-planeparallel cases, we performed test 
calculations for some typical two dimensional situations (see Sect. 3.2).

\section{Tests of the multigroup method}

This section presents the results of tests that were carried out in order to assess the performance of the multigroup method. While the systematic study by Ludwig (1992) used temperature structures of one-dimensional radiative-equilibrium atmospheres as a criterion for the quality of multigroup models, we focus on the radiative heating rate, which is the quantity that directly enters the system of (M)HD equations. In Sect. 3.1 we present one-dimensional test calculations, which aim at an optimal approximation with a small number of bins. These tests are extended to typical two-dimensional situations in Sect. 3.2, where a simplified flux-sheet model and a snapshot from a time-dependent 2D simulation are considered. In Sect. 3.3 we study the convergence properties of different opacity binning schemes in the limit of a large number of bins and present an approach for defining average opacities which can enhance the quality of the multigroup approximation. In Sect. 3.4 we give an estimate for the characteristic timescales connected with the errors occuring in multigroup calculations. Section 3.5 briefly addresses the errors resulting from the use of ODFs, and in Sect. 3.6 we discuss the influence of velocity gradients on radiative heating rates.

We treat the radiative transfer with the method of short characteristics (SC) according to Kunasz \& Auer (1988). Starting from the formal solution of the RTE (here stated for the $1 \mathrm{D}$ case)

$I_{\nu}(\tau, \mu)=I_{v}\left(\tau_{0}, \mu\right) \mathrm{e}^{-\left(\tau_{0}-\tau\right) / \mu}+\frac{1}{\mu} \int_{\tau}^{\tau_{0}} B_{v}(t) \mathrm{e}^{-(t-\tau) / \mu} \mathrm{d} t$

for a given cosine $\mu>0$ of ray-inclination and for $\tau<\tau_{0}$, the radiation transport is solved piecewise along the distance between two gridpoints. For this purpose the formal solution is rewritten for gridpoint-interval $(i, i+1)$ (the index $v$ being dropped for clarity):

$I_{i+1}(\mu)=I_{i}(\mu) \mathrm{e}^{-\Delta \tau_{i} / \mu}+\frac{1}{\mu} \int_{\tau_{i+1}}^{\tau_{i}} B(t) \mathrm{e}^{-\left(t-\tau_{i+1}\right) / \mu} \mathrm{d} t$.

In $1 \mathrm{D}$ calculations the upwind intensity $I_{i}$ has been obtained in the previous step of the computation; in two or three dimensions, the ray is traced back from point $i+1$ until it reaches a cell boundary. At the point of intersection the upwind intensity $I_{i}$ is interpolated from the values at the neighbouring gridpoints (which must be known, of course). All 2D calculations in Sect. 3.2 use linear interpolation of the upwind intensities. The physical parameters, which are given at the gridpoints, have to be interpolated along the interval in order to evaluate $\Delta \tau_{i}=\tau_{i}-\tau_{i+1}$ and $\Delta I_{i}=\int_{\tau_{i+1}}^{\tau_{i}} B(t) \mathrm{e}^{-\left(t-\tau_{i+1}\right) / \mu} \mathrm{d} t . \varrho(z)$ and $\kappa(z)$ are assumed linear in $z, B(\tau)$ linear in $\tau$, which leads to analytical expressions for $\Delta \tau_{i}$ and $\Delta I_{i}$. For all tests in planeparallel atmospheres, a Gaussian quadrature with ten directioncosines per hemisphere (Lowan et al. 1942) is used in order to discretize the angular dependence of the radiation field. The 2D calculations of Sect. 3.2 use the A8 angular quadrature of Carlson (1963) with ten ray directions per octant (see also

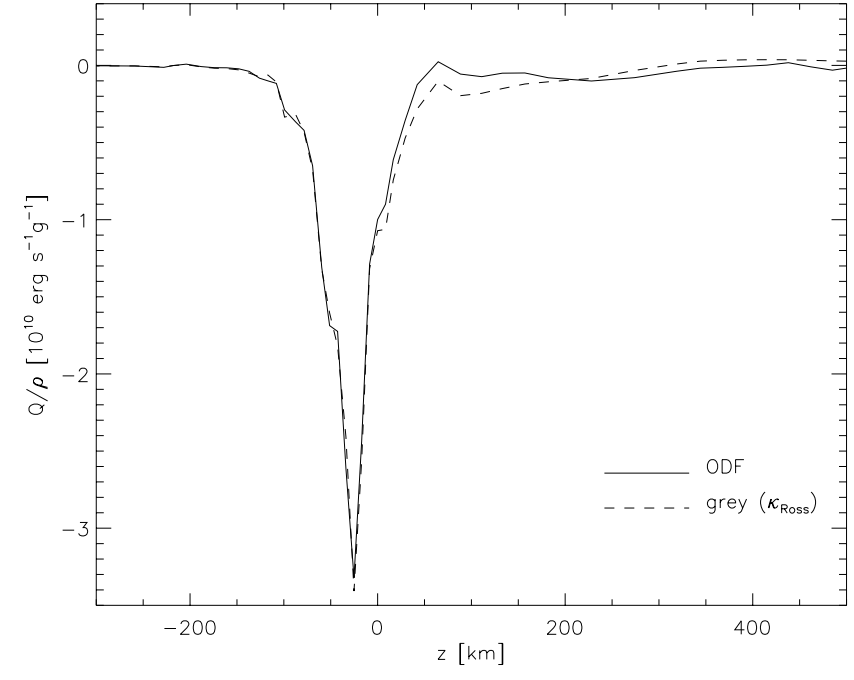

Fig. 4. The total radiative heating rate per mass unit, $Q_{\mathrm{rad}} / \varrho$ as a function of geometrical height for the 1D atmosphere of Maltby et al. (1986). The ODF solution and the grey case with the Rosseland mean opacity are shown. In the height range between $z=0$ and $z=200 \mathrm{~km}$, the line opacities lead to a backwarming effect of the ODF case relative to the grey one. $z=0$ corresponds to continuum optical depth unity at $500 \mathrm{~nm}, \tau_{500}=1$.

Bruls et al. 1999 for details about the quadrature scheme and the influence of angular resolution on $Q_{\mathrm{rad}}$ ). We use the radiative heating rates calculated with ODFs as reference solutions for evaluating the multigroup results. ODF opacitites also serve as the reference "line" spectrum on which the multigroup sorting procedure is performed. We use the ODF table for solar composition and a microturbulence parameter of $2 \mathrm{~km} \mathrm{~s}^{-1}$, which is part of the ATLAS9 stellar atmosphere package of Kurucz (1993). The ODFs cover the wavelength range from $8.98 \mathrm{~nm}$ to $10000 \mathrm{~nm}$, which is divided into $328 \mathrm{in}$ tervals. The interval size is roughly proportional to wavelength. In the most relevant spectral range $300-1000 \mathrm{~nm}$ the spectral resolution is $10 \mathrm{~nm}$. Within each of the 328 ODF intervals, the distribution function is approximated by 12 steps, i.e., the line spectrum is approximated by $328 \times 12$ "frequency points" (the frequency information is lost within each ODF interval as a result of the reordering of opacity values). The ODFs are tabulated as functions of gas pressure and temperature. The continuum opacities were extracted from the relevant routines of the ATLAS9 code (Kurucz 1993) and stored as a table on the same pressure/temperature grid as used for the ODFs.

\section{1. $1 D$ tests}

We have applied the ODF and multigroup methods to a onedimensional solar model atmosphere (Maltby et al. 1986) in order to verify the result of Ludwig (1992), who found a 5-bin description to yield a satisfactory approximation of the ODF reference solution.

The effects of a non-grey radiative transfer are illustrated in Fig. 4, which shows the frequency-integrated radiative heating rate per mass unit, $Q_{\mathrm{rad}} / \varrho$ calculated with ODFs and, for comparison, the heating rate calculated with the Rosseland mean 


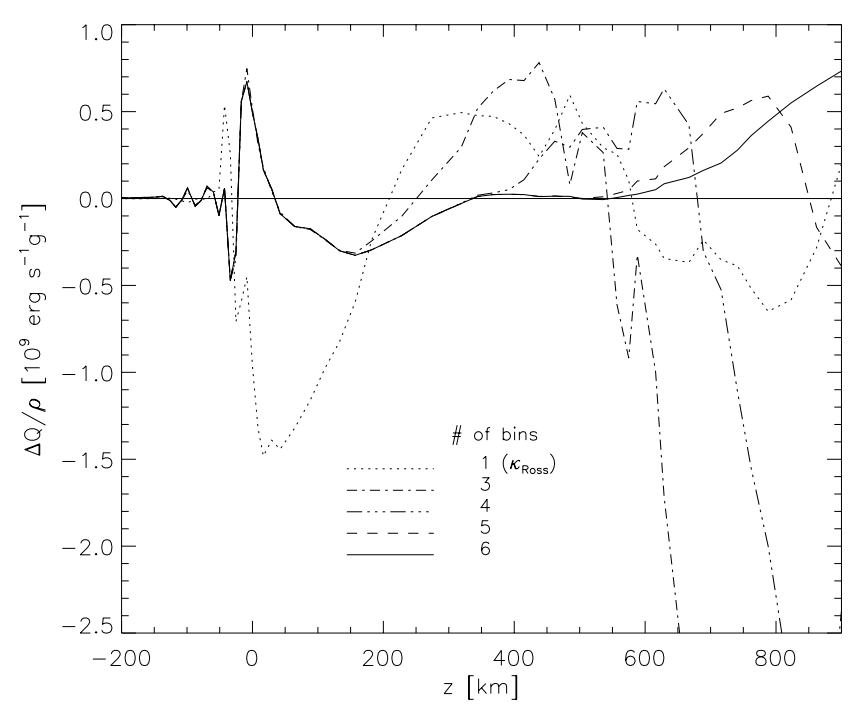

Fig. 5. $\left(Q-Q_{\mathrm{ODF}}\right) / \varrho$ as a function of height $z .5$ different cases with increasing numbers of frequency bins are shown.

opacity. Both curves show the characteristic cooling located around the height $z=0$, where the atmosphere becomes transparent in the visible continuum and where most of the emerging solar flux originates. The largest differences between the curves are found in the optically thin regions at $z>0$. In the height range $0<z<200 \mathrm{~km} Q_{\mathrm{ODF}}$ is less negative than $Q_{\text {grey }}$, which can be interpreted as the backwarming effect due to the line opacities. Above $z=200 \mathrm{~km}$ the line-cooling effect causes $Q_{\mathrm{ODF}}$ to become smaller than $Q_{\text {Ross }}$. In the optically thick part of the atmosphere $(z<0)$ the relative error of the grey solution becomes negligible as the radiative transfer approaches the diffusion limit.

Figure 5 shows $\left(Q-Q_{\mathrm{ODF}}\right) / \varrho$ for the Rosseland solution and for $\tau$-sorted multigroup models with the number of opacity bins increasing from 3 to 6 . The threshold values separating the bins in the multigroup models are spaced apart by $\Delta \log \tau_{500}=1.5$, the lowest threshold value being $\log \tau_{500}=0$ for all models, i.e. $\log \tau^{l}=-l \cdot \Delta$ with $l=0, \cdots, N-2$ and $\Delta=1.5$, where $N$ is the number of bins. This choice corresponds to a roughly equidistant spacing of levels on the geometrical height scale as well, with $\Delta z \approx 150, \cdots, 200 \mathrm{~km}$. The 3- and 4-bin models show a strong cooling above, respectively, $z \approx 550 \mathrm{~km}$ and $z \approx 700 \mathrm{~km}$. In these cases, the strong line opacities which become transparent in the upper part of the atmosphere are only crudely represented by the highest opacity bin, which results in a bad approximation of $Q_{\text {rad }}$ at large heights. In the height range $0<z<700 \mathrm{~km}$ the models with 5 and 6 bins are clearly more accurate than the grey solution. The quality of the 5- and 6-bin approximations is comparable, significant differences occur only above $z \approx 600 \mathrm{~km}$ as a result of the additional threshold of the 6-bin model at $\log \tau_{500}=-6$ (corresponding to $z \approx 760 \mathrm{~km}$ ). Likewise, the 3 - and 4-bin solutions differ from the 6-bin model only in the height range above, respectively, $z \approx 150 \mathrm{~km}$ and $z \approx 400 \mathrm{~km}$, corresponding that parts of the atmosphere which are covered by the respective highest bins of the 3- and 4-bin models.

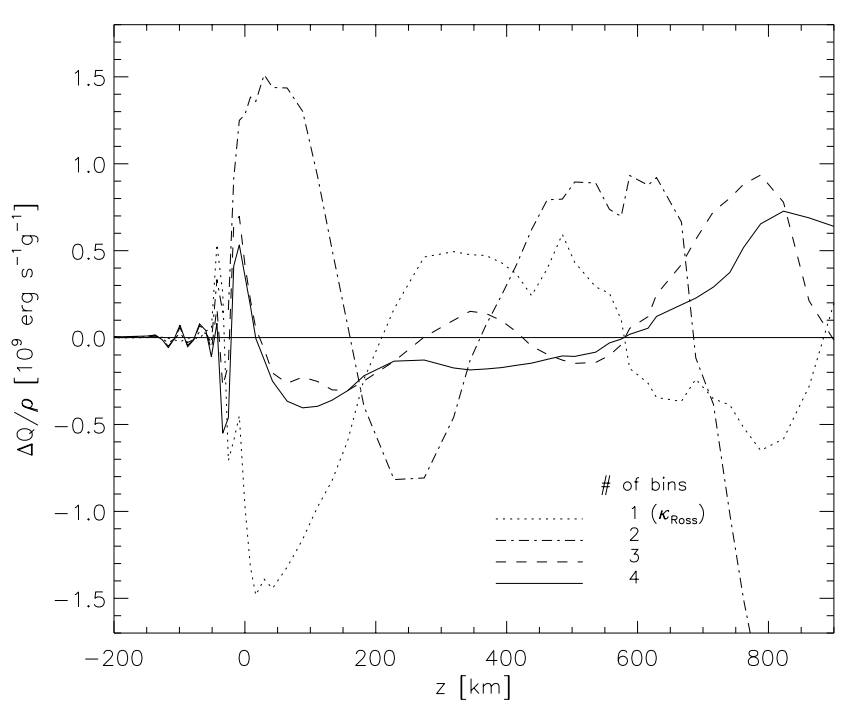

Fig. 6. $\left(Q-Q_{\mathrm{ODF}}\right) / \varrho$ as a function of height $z .4$ different cases with increasing numbers of frequency bins are shown.

Figure 6 shows the attempt to model $Q_{\text {rad }}$ with a small number of bins distributed equidistantly over the height range $z>0$. The threshold values (in $\log \tau_{500}$ ) were chosen as follows:

2 bins: -3

3 bins: $-1.5,-4.5$

4 bins: $-1,-3,-5$.

It turns out that the 2-bin model is less accurate than the Rosseland solution. Obviously, switching from the Rosseland to the Planck mean results in a bad representation of opacities in cases where the number of bins is small and the classification of frequencies is accordingly crude. While the 3-bin solution shows a relatively large error above $z \approx 600 \mathrm{~km}$, the performance of the 4-bin solution is comparable to the cases with 5 and 6 bins in Fig. 5. Calculations with larger numbers of equidistantly spaced bins did not show any significant further improvement (see Sect. 3.3). So, for the atmosphere model considered here, the optimal number of frequency bins is found to be 4-6. However, as the results of Fig. 5 suggest, the optimal number depends on the vertical extent of the atmosphere considered.

The relatively large errors around $z=0$ do not decrease with increasing number of bins. As mentioned in Sect. 2.2, one does not expect perfect convergence towards the exact solution, and it is plausible that the large gradients of opacity and source function around $z=0$ lead large absolute errors in $Q_{\text {rad }}$. However, since this is the region where strong radiative cooling leads to large absolute values of $Q_{\text {rad }}$ itself, the relative errors in $Q_{\text {rad }}$ near $z=0$ are small and do not seriously impair the quality of the multigroup solutions (see Sect. 3.4 for an estimate of the importance of errors in $Q_{\text {rad }}$ ). In the optically thick regime $\tau_{500}>1(z<0)$ the solutions for different total numbers of bins agree well with each other, the relative error $\left|\left(Q-Q_{\mathrm{ODF}}\right) / Q_{\mathrm{ODF}}\right|$ not exceeding the order of $10^{-2}$. This is in agreement with the fact that the frequency dependence has almost no influence on the radiative transfer at large optical depths and any multigroup model can be expected to yield 

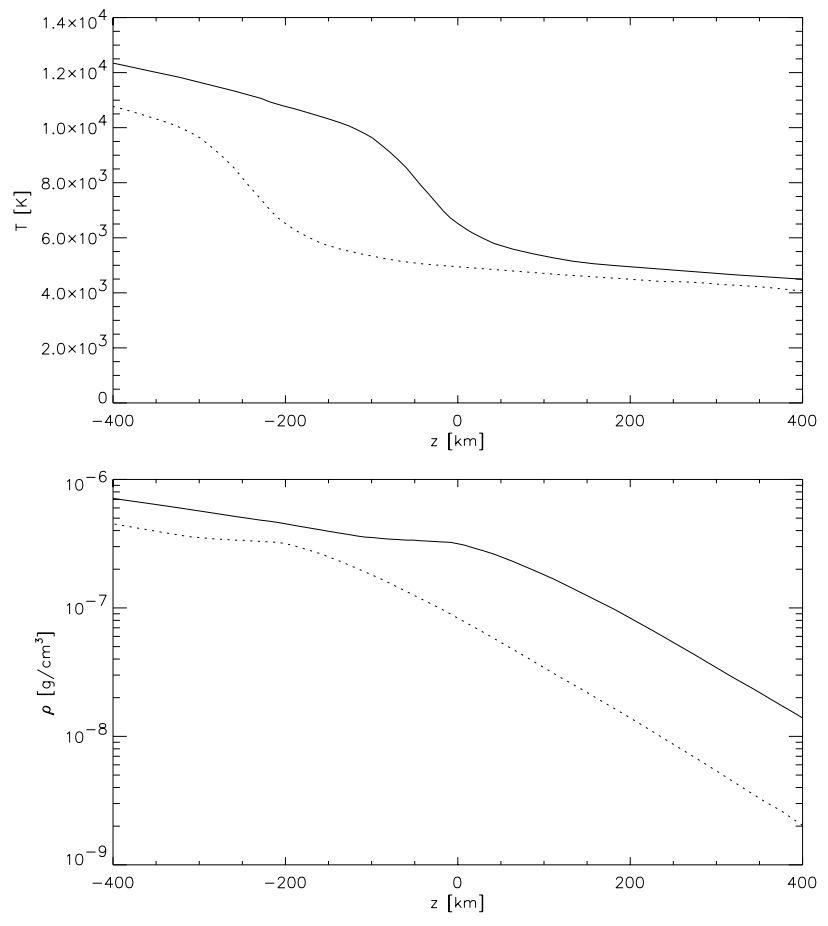

Fig. 7. Temperature and density on the symmetry axis (center) of the magnetic sheet (dotted) and in the surrounding atmosphere (solid).

good results at these depths as long as the Rosseland mean is used for calculating bin-averaged opacities.

\subsection{Results of $2 D$ calculations}

The previous section has shown that, in a plane-parallel atmosphere, a substantial improvement of the radiative heating rates in comparison to the grey case can be achieved already with a moderate number of frequency bins. In this section, we consider two-dimensional situations in order to assess the error incurred by using a $1 \mathrm{D}$ reference atmosphere in the $\tau$-sorting procedure. For multigroup calculations, the same bin parameters as in the previous section have been used.

As a first step we investigated a simple model of a 2D magnetic flux sheet. Based on the 1D atmosphere used in the previous section, the stratification in the interior of the sheet is shifted downwards by $200 \mathrm{~km}$ relative to the surrounding atmosphere (the "Wilson depression"; see Fig. 7, right panels). The width of the sheet as a function of height results from magnetic flux conservation together with the condition of total (magnetic + gas) pressure equilibrium between the interior and the exterior of the sheet. The equilibrium condition reads

$\frac{B_{\text {in }}^{2}(z)}{8 \pi}=\Delta p(z)$,

where $B_{\text {in }}$ is the field strength in the sheet and $\Delta p=p_{\mathrm{ex}}-$ $p_{\text {in }}$ is the difference between external and internal gas pressure. Substituting $B_{\text {in }}(z)$ from the above equation in the expression for flux conservation

$B_{\text {in }}(z) w(z)=B_{\text {in }}(0) w(0)$
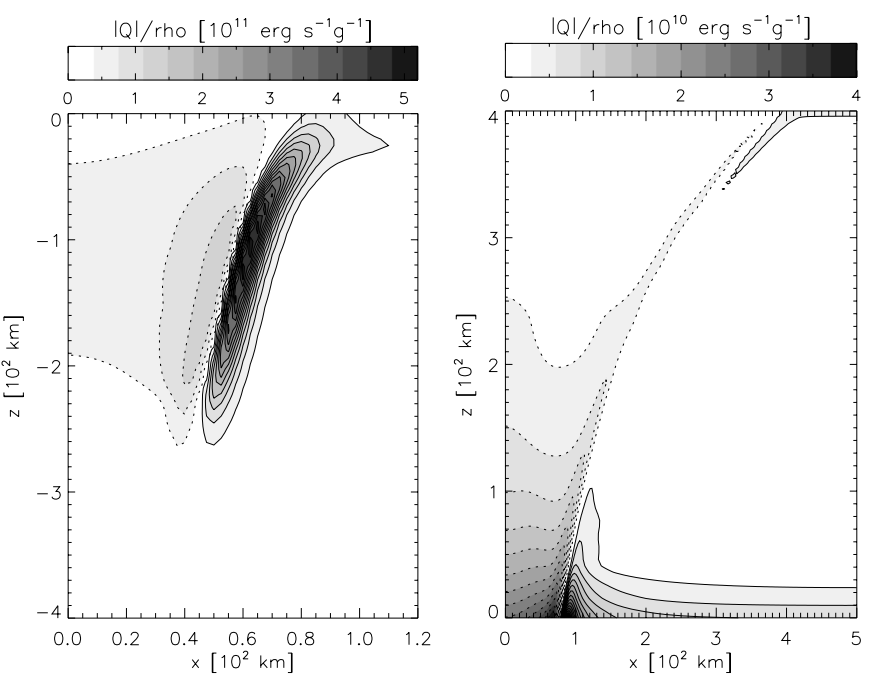

Fig. 8. $Q_{\text {rad }} / \varrho$ for the ODF-based reference solution in a magnetic flux sheet model. The left and right panels show the lower part $(z<0)$ and upper part $(z>0)$ of the sheet, respectively. Solid contour lines indicate cooling $(Q<0)$, dashed lines indicate heating $(Q>0)$.

( $w(z)$ is the width of the sheet), one obtains

$w(z)=\sqrt{\frac{\Delta p(0)}{\Delta p(z)}} w(0)$.

At the height $z=0$, corresponding to $\tau_{500}=1$ in the exterior, the flux sheet has a width of $150 \mathrm{~km}$. The sheet is fanning out with increasing height as the magnetic pressure necessary to balance the jump in gas pressure decreases (see Fig. 7, left panel). At the interfaces between the interior and the exterior of the sheet, the atmospheric parameters are smoothed out horizontally over a distance of several tens of kilometers:

$T(x)=T_{\mathrm{in}}+\left(T_{\mathrm{ex}}-T_{\mathrm{in}}\right) \cdot \frac{1}{2}\left[\operatorname{ERF}\left(\frac{x-x_{\mathrm{b}}}{\Delta x}\right)-1\right]$,

and likewise for pressure and density; $x_{\mathrm{b}}$ is the horizontal position of the sheet boundary, for the smearing parameter $\Delta x$ we used a value of $10 \mathrm{~km}$. Owing to the mirror symmetry of the sheet, the calculations can be restricted to one half of the flux sheet, with symmetrical boundary conditions imposed on the sheet's symmetry axis (located at $x=0$ ). A cartesian grid with $201 \times 161$ grid points and horizontal and vertical resolutions of, respectively, $2.5 \mathrm{~km}$ and $5 \mathrm{~km}$ was used.

In Fig. 8, the heating rates for the ODF reference solution are shown. For $z>-200 \mathrm{~km}$, the sheet interior is heated as a result of the lateral radiative energy exchange with the hotter exterior. Correspondingly, the outside regions close to the sheet boundary are subject to strong cooling. The weak cooling, which occurs below $z=-200 \mathrm{~km}$ inside the sheet around the symmetry axis, is caused by radiative energy losses as the interior becomes optically thin at these heights. Figure 9 shows $Q_{\text {rad }}$ along horizontal cuts through the (half) sheet at three different heights. At $z=-150 \mathrm{~km}$, the agreement between the ODF reference and both the grey and multigroup solutions is very good. Corresponding to the horizontal temperature gradient, a heating peak inside the sheet and a stronger cooling region outside form near the sheet boundary. At this 

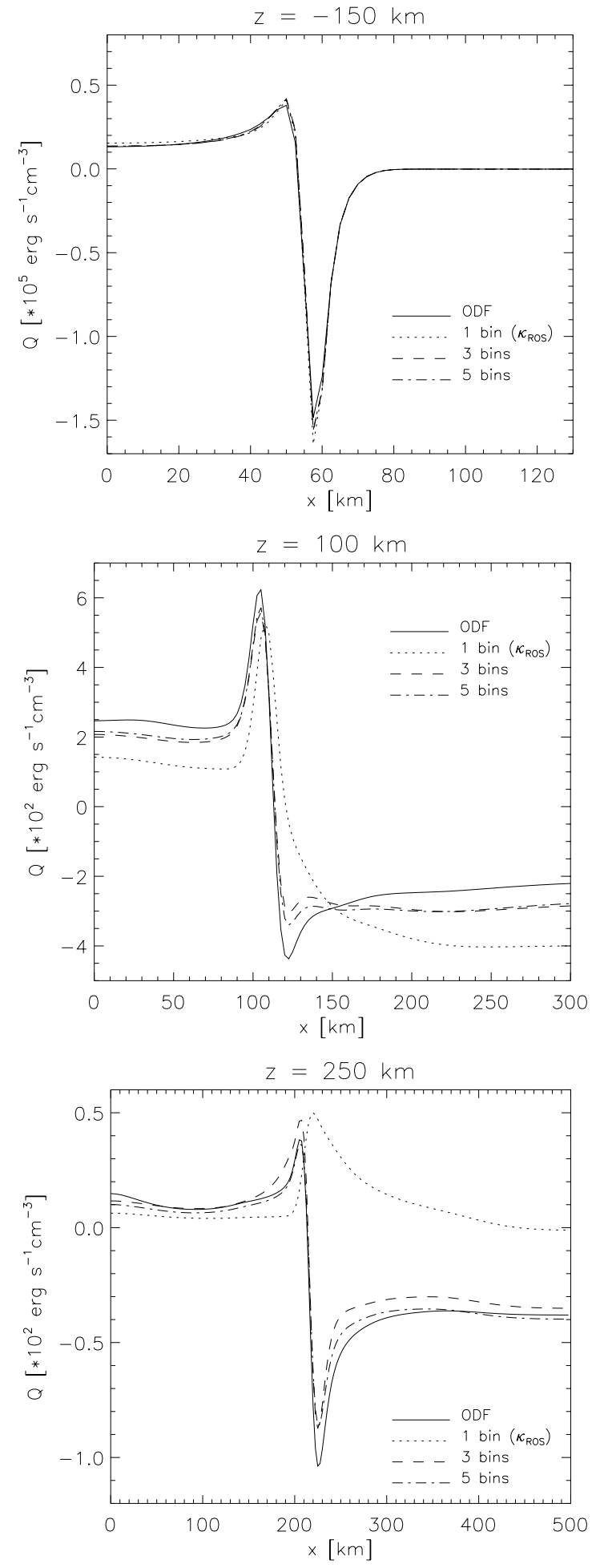

Fig. 9. Horizontal profiles of $Q_{\text {rad }}$ at three different heights in the 2D atmosphere with an embedded flux sheet. $x=0$ corresponds to the symmetry axis of the sheet.

height, the outside atmosphere and most of the boundary region are optically thick and the radiative transfer is essentially grey, so that $Q_{\text {rad }}$ is well represented even without a detailed treatment of the frequency dependence. The maximum relative errors near the heating and cooling peaks range between 5 and 10 percent.
At $z=100 \mathrm{~km}$, both multigroup solutions qualitatively reproduce the ODF case. The grey (Rosseland) case is much less accurate than the 3- and 5-bin solutions. The heating peak at the boundary is shifted outwards by approximately $10 \mathrm{~km}$, the peak value being reduced by 15 percent with respect to the ODF case, while the small cooling dip outside is not captured at all. The relative errors of the multigroup solutions are largest at the cooling dip around $x=120 \mathrm{~km}$ with 30 and 20 percent for the 3- and 5-bin solutions, respectively. At the heating peak $(x=105 \mathrm{~km})$ the errors do not exceed 10 percent. At a still greater height of $250 \mathrm{~km}$, both the 3- and 5-bin approximations yield acceptable results, though neither captures the full extent of the cooling peak outside the sheet boundary. Around the heating peak $(x=200 \mathrm{~km})$ and in the outside region $(x>250 \mathrm{~km})$, the 5-bin solution achieves a clear improvement over the 3-bin case. The error of the grey solution is large by comparison, and the ODF solution is not even approximately reproduced. The heating peak inside the sheet has vanished; instead, heating takes place immediately outside the sheet, where the correct solution shows considerable cooling. This behaviour can be explained by the fact that, on the basis of the Rosseland mean, the interior of the sheet is transparent at a height of $250 \mathrm{~km}$; accordingly, the interaction between gas and radiation is very weak within the sheet, resulting in small values of $Q_{\text {rad }}$. In the optically thicker regions immediately outside the sheet, radiation originating from deeper, hotter regions at the opposite sheet boundary and crossing the sheet almost unattenuated, leads to a net heating effect. The error of the grey solution far outside the sheet $(x=500 \mathrm{~km})$ is in agreement with the results of the $1 \mathrm{D}$ calculations at the same height in the atmosphere; this is plausible since far away from the sheet the radiative transfer is essentially one-dimensional. This example clearly demonstrates how important a nongrey approach to radiative transfer can be in optically thin regions in order to obtain accurate values of $Q_{\text {rad }}$.

As a further step towards more realistic situations we tested the opacity binning models in calculations that used a snapshot from a 2D simulation of granulation by Ploner et al. (1999) (Fig. 10), which has no relation to the one-dimensional reference atmosphere used for $\tau$-sorting. The vertical and horizontal extent of the computational domain is $1400 \times 1400 \mathrm{~km}^{2}$ with a grid resolution of $35 \mathrm{~km}$. Figure 11 shows $Q_{\mathrm{rad}}$ in horizontal cuts at heights of approximately 100 and $500 \mathrm{~km}$ above $\tau_{500}=1$, respectively. The same $\tau$-sorted opacity-tables as in the previous calculation have been used. In accordance with the results for the flux sheet, all solutions (including the grey case) agree quite well with each other at the lower level, although the errors of the grey case become more pronounced towards the horizontal boundaries of the domain. The differences between the 3- and 5- bin solutions are only marginal. At $500 \mathrm{~km}$ above $\tau_{500}=1$, the Rosseland solution, similar to the optically thin case in Fig. 9, completely fails to reproduce the ODF reference solution. On the other hand, the 5-bin solution excellently agrees with the reference curve and is clearly superior to the 3-bin model.

In summary, the $\tau$-sorted 5-bin model apparently yields good approximations of $Q_{\text {rad }}$ to the quasi-exact ODF case over a large height range in "realistic" 2D atmospheres. 

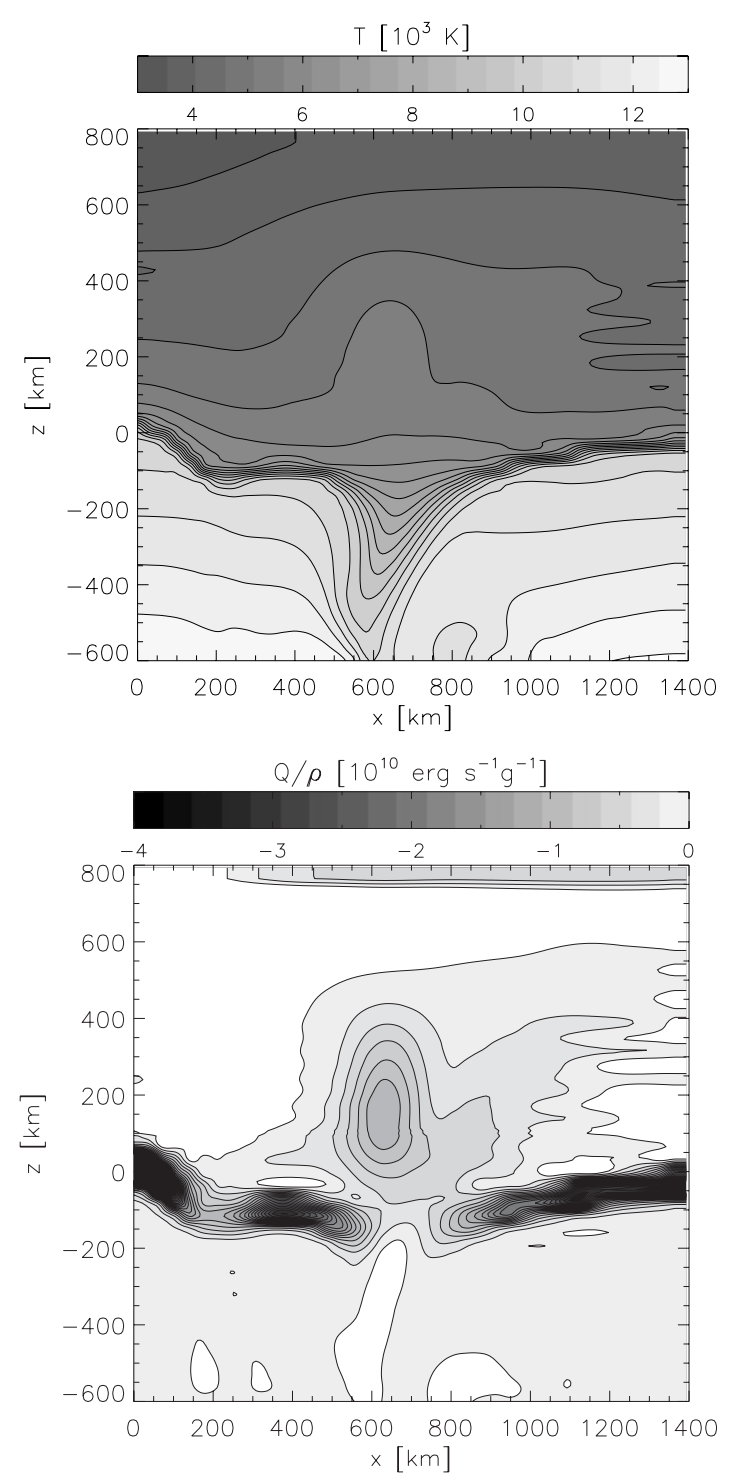

Fig. 10. Upper panel: temperature plot of a $1.4 \times 1.4 \mathrm{Mm}^{2}$ cut-out from a two-dimensional simulation snapshot (Ploner et al. 1999). Lower panel: the corresponding ODF-based radiative heating rate.

The advantage over a grey radiative transfer based on the Rosseland mean is most pronounced at large photospheric heights where spectral lines are the dominant opacity sources.

\subsection{The multigroup method in the limit of a large number of bins}

While the 5-bin $\tau$-sorting scheme shows acceptable results for computing radiative heating rates in both $1 \mathrm{D}$ and $2 \mathrm{D}$, one might ask whether any further improvement can be achieved by increasing the number of frequency bins. Full (M)HD simulations with more than a handful of bins are not feasible with the computing power available today, but with increasing computational resources, this question may become relevant in the future. In this section we study the performance of $\tau$-sorting and also $\kappa$-sorting in multigroup models with increasing numbers of bins.
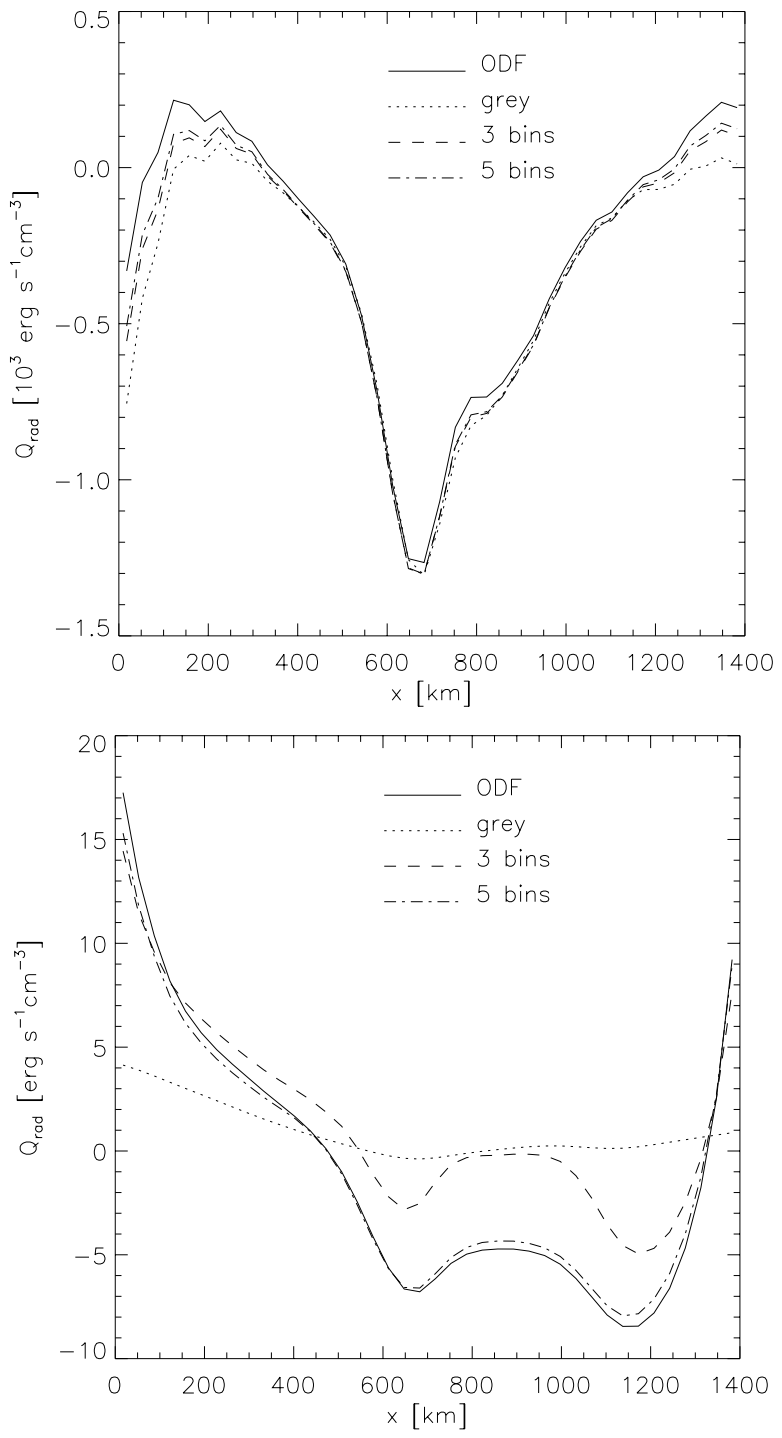

Fig. 11. Horizontal profiles of $Q_{\text {rad }}$ for a snapshot from a 2D simulation, at heights of $100 \mathrm{~km}$ (upper panel) and $500 \mathrm{~km}$ (lower panel) above $\tau_{500}=1$.

We first applied the $\tau$-sorting procedure to a high-resolution 1D atmosphere with $\Delta z=0.5 \mathrm{~km}$, which was interpolated from the reference stratification used in the previous sections. Figure 12 shows the deviation of the radiative heating rate per mass unit from the reference solution, $\left(Q-Q_{\mathrm{ODF}}\right) / \varrho$, as a function of height. The 5-bin solution uses the parameters specified above, while for larger numbers of bins the optical depthlevels separating the bins are spread evenly on the $\log \tau_{500}$-scale across the upper part of the atmosphere, $\tau_{500}<1$. The 5 -bin solution gives the best result for $z \approx 200 \mathrm{~km}-600 \mathrm{~km}$, while the error increases for greater heights. For increasing numbers of bins, the solutions seem to converge towards a limiting solution, which, however, clearly deviates from $Q_{\text {ODF }}$. This does not come as a surprise as the multigroup approach, on theoretical grounds, can not be expected to reproduce the exact solution, even in the limit of infinitely large bin numbers (see the remark at the end of Sect. 2.2). In Fig. 13, the error rate is plotted for $k$-sorted bins. As the reference optical depth (on 


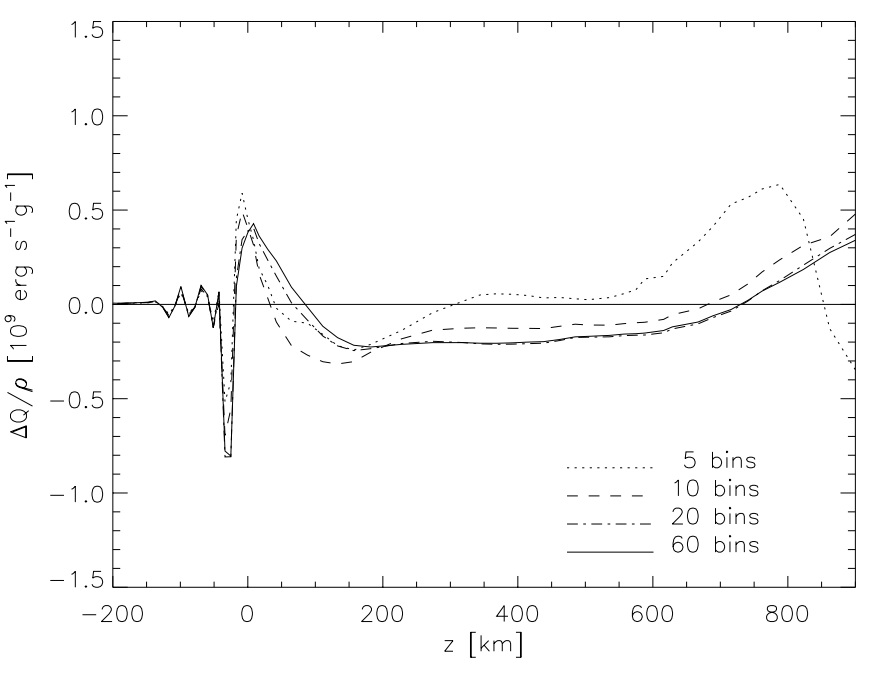

Fig. 12. Deviations of the radiative heating rate per mass unit, $Q_{\mathrm{rad}} / \varrho$, from the ODF reference solution as a function of height. Shown are results for $\tau$-sorted models with different numbers of bins.

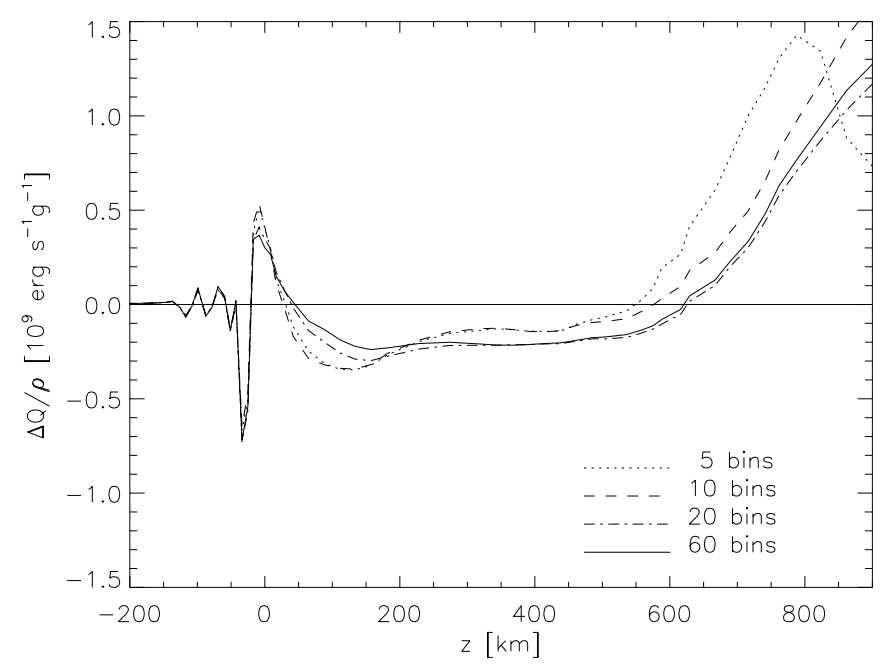

Fig. 13. Same as in Fig. 12, but for $\kappa$-sorting and a sorting parameter of $\tau_{\text {ref }}=0.1$.

the $\tau_{500}$-scale), for the opacity spectrum $\kappa_{v}\left(\tau_{\text {ref }}\right)$ on which sorting is based, we chose $\tau_{\text {ref }}=0.1$ (Ludwig 1992). In heights above $200 \mathrm{~km}$, the 5-bin solution is clearly worse than the corresponding result for the 5-bin $\tau$-sorted model. For larger numbers of bins, a pronounced advantage of the $\tau$-sorted solutions is found only above $700 \mathrm{~km}$. A different picture is obtained when using $\tau_{\text {ref }}=0.01$ (Fig. 14). Here, for heights below $600 \mathrm{~km}$ the approximation is better than in the $\tau$-sorted case. Again, as for $\tau$-sorting, the 5-bin solution gives the best overall result.

All results shown so far, the $\tau$-sorted model as well as the two $\kappa$-sorted ones, show a clear trend towards too strong heating in the uppermost regions $(z \gtrsim 700 \mathrm{~km})$. This behaviour is caused by using the Planck mean in the optically thin regime. In the regions $z \gtrsim 700 \mathrm{~km}$ we have $J_{v}>B_{v}$ for a large part of the spectrum, since for the optically thin frequencies $J_{v}$ is determined by radiation coming from deeper and hotter regions around $\tau_{v}=1$ (see Fig. 15 for an example). Consequently, $Q_{\text {rad }} \propto \int \kappa_{v} \varrho\left(J_{v}-B_{v}\right) \mathrm{d} v>0$, i.e. some

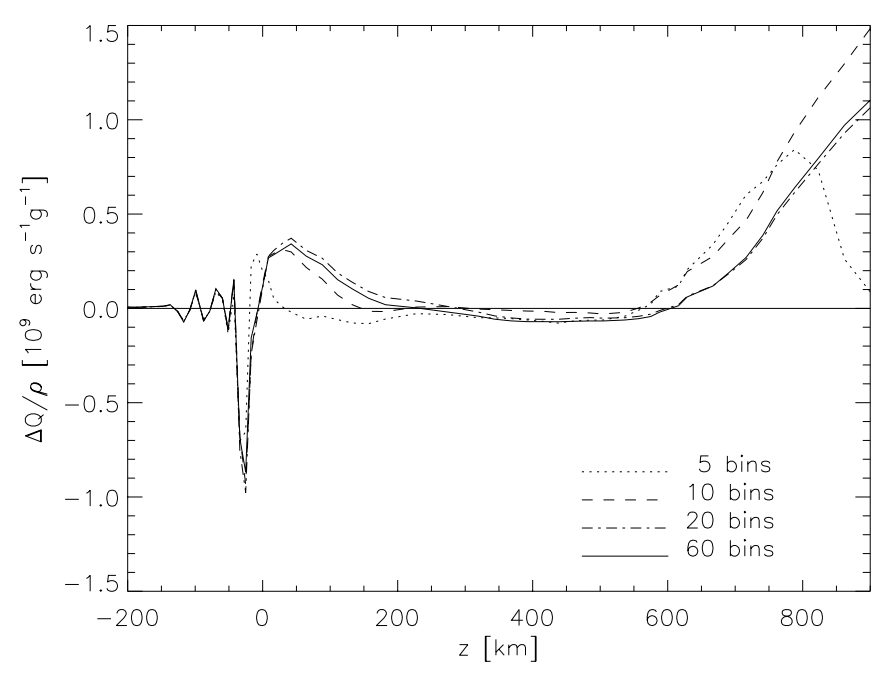

Fig. 14. Same as in Fig. 13, for $\tau_{\text {ref }}=0.01$.

heating results from the absorption of the "hot" radiation. As the Planck mean tends to overestimate the line opacities, the factor $\kappa_{\mathrm{bin}} \varrho$ in the expression for $Q_{\mathrm{rad}}$ in opacity binning models becomes too large and the heating in the optically thin regions is overestimated. We have seen that this effect is more pronounced for the $\kappa$-sorted models. This may be explained by the fact that $\kappa$-sorting does not properly account for the line opacities above $\tau_{\text {ref. }}$ As a consequence, in regions above $\tau_{\text {ref }}$, opacities of considerably varying magnitude may be collected in a given bin, resulting in a bad representation of opacities. This is not the case when $\tau$-sorting is used, since here the integral $\int_{0}^{z(\tau=1)} \kappa_{\nu} \varrho \mathrm{d} z$ contributes to the sorting process. Thus, a systematic advantage of $\tau$-sorting over $\kappa$-sorting is found only for heights above $700 \mathrm{~km}$. The quality of the 5-bin solution, both for $\tau$-sorting and for $\kappa$-sorting, depends on the particular choice of the bin levels, so the results shown in Figs. 12 and 14 do not necessarily imply that a small number of bins always yields the best approximation.

As the systematic overestimation of the heating in the uppermost parts of the atmosphere is caused by the Planck mean, it might be worthwhile to look for a possible replacement which performs better in the optically thin regime. As already mentioned in Sect. 2, the derivation of the Planck mean is based on the assumption $J_{v} \approx B_{v} / 2$ (implying $J_{v}-B_{v} \approx-B_{v} / 2$ ), which is strictly correct only for the optically thin parts of an isothermal atmosphere (the factor $1 / 2$ accounting for the fact that the uppermost parts of the atmosphere are only illuminated from the hemisphere underneath). However, upon inspection of Fig. 15, which shows $J_{v}$ and $B_{v}$ for the ODF-calculation at the top of the reference atmosphere, it becomes immediately clear that this relation does not even approximately hold at great heights in the atmosphere.

In order to derive an alternative prescription for a bin average at small optical depths, we set out from Eq. (7) which gives the general definition of a bin-averaged opacity. Instead of replacing the weighting function $J_{v}-B_{v}$ with $B_{v}$, one might try to obtain $J_{v}-B_{v}$ by using the exact radiation field $J_{v, \mathrm{ODF}}$ at the top of the atmosphere at $\tau=0$, which results from an 


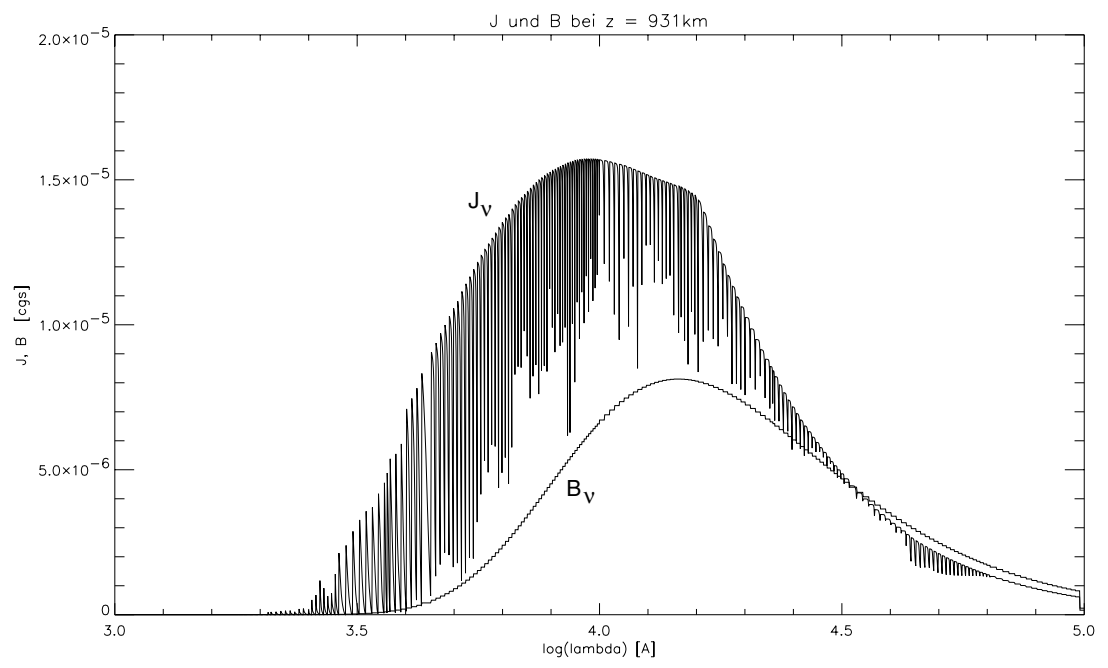

Fig. 15. $J_{v}$ from an ODF-calculation and $B_{v}$ (lower curve) at the top $(z=931 \mathrm{~km})$ of the reference atmosphere. At this height the atmosphere has a temperature of $3500 \mathrm{~K}$.

ODF reference calculation (plotted in Fig. 15). Two problems immediately arise:

(i) the opacity tables that result from the multigroup approach must be applicable to atmospheres different from the reference stratification. The intensity spectrum $J_{v, \mathrm{ODF}}(\tau=0)$ may be correct for the optically thin regions of the reference atmosphere, but there is no guarantee that it is a good approximation in the higher regions of other atmospheres. On the other hand, the $\tau$-sorting approach requires a reference atmosphere anyway, and there is no reason why the properties of this particular atmosphere should have an influence on the frequency-sorting only;

(ii) the weighting function $J_{v}-B_{v}$ is not positive definite. Therefore, straightforward application of the averaging procedure may lead to negative mean opacities.

Dividing the frequency bin into those frequencies where $J_{v}-$ $B_{v} \geq 0$ and those where $J_{v}-B_{v}<0$, one obtains

$$
\begin{aligned}
Q_{\text {bin }} / 4 \pi \varrho & =\int_{+} \kappa_{v}\left(J_{v}-B_{v}\right) \mathrm{d} v+\int_{-} \kappa_{v}\left(J_{v}-B_{v}\right) \mathrm{d} v \\
& =\kappa^{+} \int_{+}\left(J_{v}-B_{v}\right) \mathrm{d} v+\kappa^{-} \int_{-}\left(J_{v}-B_{v}\right) \mathrm{d} v
\end{aligned}
$$

with the positive-definite averages

$\kappa^{+}=\frac{\int_{+} \kappa_{v}\left(J_{v}-B_{v}\right) \mathrm{d} v}{\int_{+}\left(J_{v}-B_{v}\right) \mathrm{d} v}$

and

$\kappa^{-}=\frac{\int_{-} \kappa_{v}\left(J_{v}-B_{v}\right) \mathrm{d} v}{\int_{-}\left(J_{v}-B_{v}\right) \mathrm{d} v}$.

If $\kappa^{+}$and $\kappa^{-}$are of the same order, we may define

$\bar{\kappa}=\frac{1}{2}\left(\kappa^{+}+\kappa^{-}\right)$

in cases, where straightforward use of $J_{v, \mathrm{ODF}}-B_{v}$ as the weighting function leads to negative results, since then

$Q_{\text {bin }} / 4 \pi \varrho \approx \frac{1}{2}\left(\kappa^{+}+\kappa^{-}\right) \int_{\text {bin }}\left(J_{v}-B_{v}\right) \mathrm{d} v$

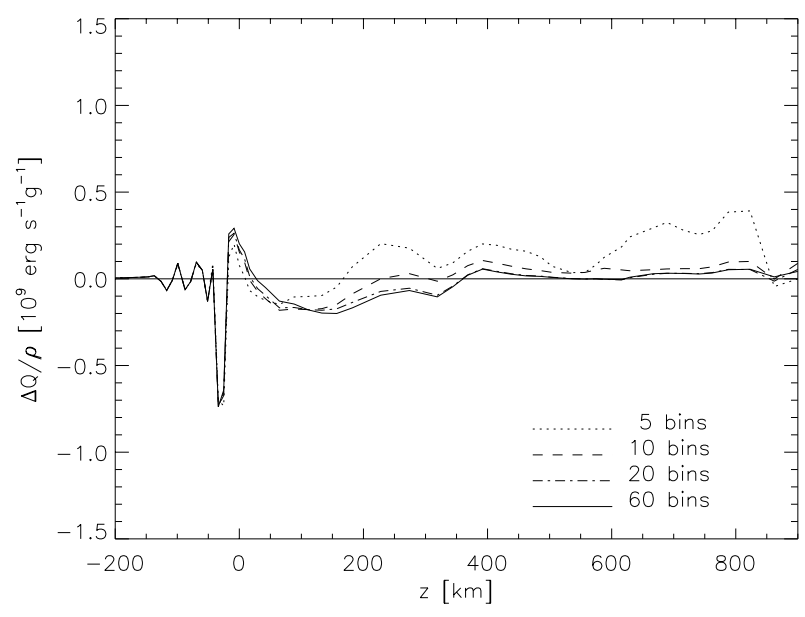

Fig. 16. Same as Fig. 12, for a $\tau$-sorted model where the Planck mean has been replaced in optically thin regions by the $J-B$ mean described in the text.

and the heating rate is, at least approximately, recovered.

Figure 16 shows the results of test calculations with the averaging procedure using $J_{v, \mathrm{ODF}}(\tau=0)-B_{v}$ as weighting function. A transition depth $\tau_{0}=0.1$, below which the Rosseland mean is used, has been chosen. $\tau$-sorting has been applied, where now those frequencies are grouped together, which reach $\tau_{v}=0.5$ at the same height-level. Whenever a negative opacity-value occured it was replaced by expression (28).

The results shown in Fig. 16 indicate that the new averaging appears to work well for calculations with 10 or more bins. Here the agreement with the ODF-solution is excellent throughout the whole height range, including the regions $z>$ $700 \mathrm{~km}$. The quality of the 5 bin solution, however, is not that impressive, and while the accuracy of the 5-bin approximation depends rather sensitively on the particular values of the bin levels, we could not achieve a significant improvement with different choices of levels.

The advantage of the new method in optically thin regions is still observable in atmospheres, which differ strongly from 


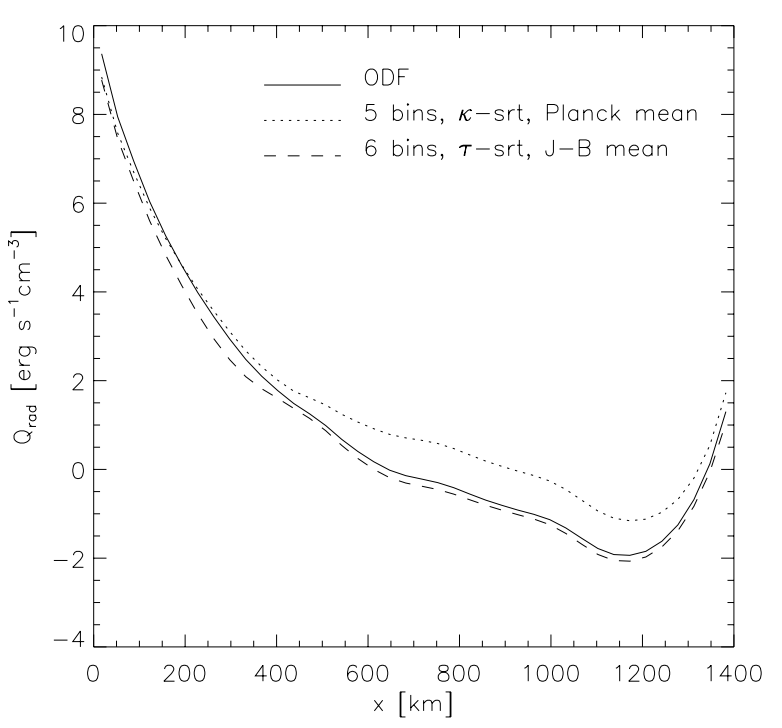

Fig. 17. $Q_{\text {rad }}$ for a horizontal cut through a $2 \mathrm{D}$ snapshot at a height of approx. $700 \mathrm{~km}$ above $\tau_{500}=1$. For $x>400 \mathrm{~km}$ the $J-B$ mean improves the quality of the approximation.

the reference atmosphere. Figure 17 shows a horizontal cut at a height of approx. $700 \mathrm{~km}$ above $\tau_{500}=1$ through the $2 \mathrm{D}$ simulation snapshot used in Sect. 3.2. The solution obtained by using the $J-B$ mean is clearly closer to the reference solution for $x>400 \mathrm{~km}$ than the $\kappa$-sorted 5 bin solution using the Planck-mean.

In principle, the new opacity averaging procedure seems to be superior to the use of the Planck-mean when a sufficiently large number of bins is used. This is particularly true for simulations which are extended to include chromospheric layers, since at these heights the advantage is most pronounced. Since 2D/3D simulations are presently restricted to multigroup calculations with 4 or 5 bins at best, the merits of the new averaging procedure appear to be rather academic. The improvement due to the modified average may, however, become relevant in the future, when the increase of computing power makes simulations with a larger number of bins feasible.

\subsection{Error timescales}

Having compared the accuracy of different opacity binning models, it seems worthwhile to obtain an estimate of the importance of the errors which result from the various approaches. Given the results of the previous sections, two questions are of particular interest:

(i) Does the error of the grey solution make a nongrey treatment mandatory?

(ii) How severe is the systematic error at large heights $(z>$ $600 \mathrm{~km}$ ) introduced by the Planck mean?

As an indicator for the severity of the error we use the time scales on which the deviation from the reference $Q_{\mathrm{ODF}}$ affects the temperature structure of the atmosphere. In order to assess how important the error is, it is plausible to compare these scales with typical dynamical time scales in the photosphere, which give an estimate for the time span over which a volume element of the flowing plasma is exposed to the inaccuracies of $Q_{\text {rad }}$ in a simulation with grey or multigroup radiative transfer.

In order to isolate the effect of the error in $Q$, we assumed that the $1 \mathrm{D}$ reference atmosphere is kept in radiative equilibrium by a time-independent source term $Q_{\mathrm{s}}$ that represents the necessary non-radiative heating or cooling, i.e.

$Q_{\mathrm{ODF}}(z)+Q_{\mathrm{s}}(z) \equiv 0$.

Any heating or cooling is then solely caused by the error $\Delta Q=$ $Q_{\text {app }}-Q_{\text {ODF }}$ introduced by the approximative treatment of the frequency dependence. Starting with the unperturbed temperature stratification $T_{0}(z)$ for $t=0$, the time development of the temperature is assumed to be given by

$\frac{\partial T}{\partial t}(z, t)=\frac{\Delta Q(z, t)}{c_{V} \varrho}$

with $\Delta Q(z, t)=Q_{\text {app }}(z, t)-Q_{\mathrm{ODF}}(z, t=0)$, allowing the temperature to relax to a new equilibrium $T_{\text {eq }}(z)$ for which

$\left.\Delta Q(z)\right|_{\mathrm{eq}}=\left.Q_{\mathrm{app}}(z)\right|_{\mathrm{eq}}-Q_{\mathrm{ODF}}(z, t=0) \equiv 0$

or

$\left.Q_{\mathrm{app}}(z)\right|_{\mathrm{eq}}+Q_{\mathrm{s}}(z) \equiv 0$.

holds.

Equation (31) was solved by explicit forward integration in time. When computing $Q_{\mathrm{app}}(z, t)$ for each timestep, the changes of $p(z)$ and $\varrho(z)$ required by the condition of hydrostatic equilibrium have been neglected, so our calculation can be expected to give reasonable results only as long as the relative change in temperature remains small. Figure 18 shows the time development of the temperature stratification for the Rosseland approximation (top), the 5-bin $\tau$-sorted model used in Sects. 3.1 and 3.2 (middle), and a 20-bin $\tau$-sorted model for which the Planck mean has been replaced by the $J-B$ mean described in the previous section (bottom). In the grey case, a strong cooling occurs below $z=0$, which extends to deeper layers with increasing time. An even more drastic change in temperature occurs above $500 \mathrm{~km}$, where the temperature drop exceeds $10^{3} \mathrm{~K}$ after a few minutes. However, here the temperature runs out of the range, for which the violation of hydrostatic equilibrium is tolerable. Using the photospheric pressure scale height of about $150 \mathrm{~km}$ as characteristic length scale and assuming typical flow velocities of $1-2 \mathrm{~km} \mathrm{~s}^{-1}$, one obtains a dynamical time scale of the order of $10^{2} \mathrm{~s}$. So, the Rosseland mean produces temperature disturbances of several hundred $\mathrm{K}$ on time scales that are comparable to typical dynamical time scales. Therefore, one expects these disturbances to have a significant effect on the temperature structure. For the 5-bin case (middle panel), the situation is entirely different. Above $z=0$ the temperature stratification settles to a new equilibrium within $30 \mathrm{~s}$. The deviation of about $60 \mathrm{~K}$ from the initial state however is much smaller than in the grey case and should not have serious consequences for the physics in the atmosphere. For $z<0$, the reaction of the atmosphere is much slower, owing to the diffusive character of radiative transfer in the optically thick regime. New equilibrium values for the temperatures have not 

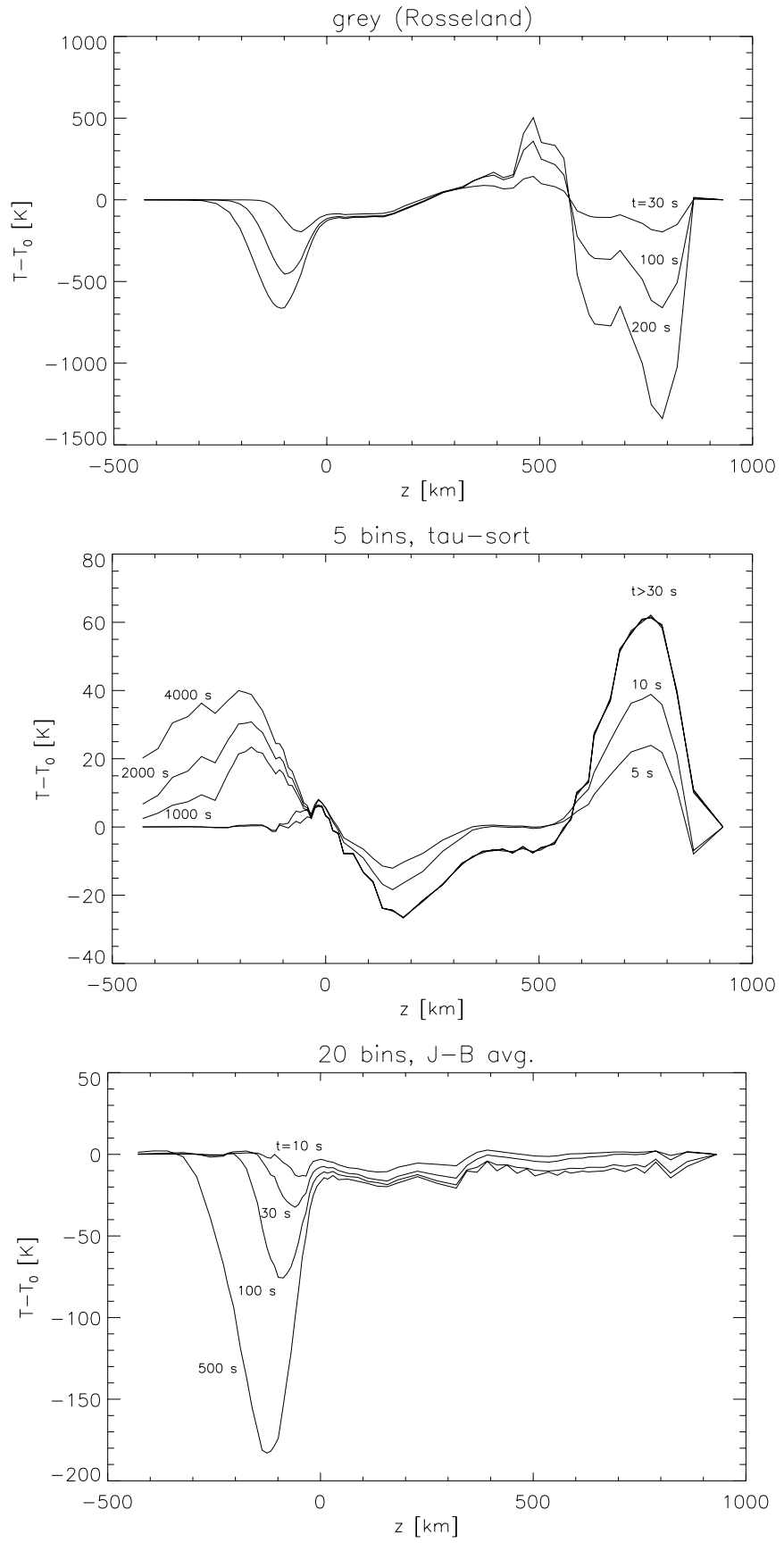

Fig. 18. Change in temperature for different times after the calculations started. The three panels refer to three different opacity binning models described in the text. Note the different ordinate scales for each panel.

been reached after $4 \times 10^{3} \mathrm{~s}$ simulated time and the changes in temperature that can be achieved on dynamical timescales are negligible.

The lower panel of Fig. 18 shows that replacing the Planck mean with the $J-B$ averaging procedure further reduces the temperature disturbance for $z>0$, but considering the results for the 5-bin model, the Planck mean still seems to be an acceptable choice. Below $z=0$ the impact on the temperature is stronger but remains smaller than $100 \mathrm{~K}$ within a time of $10^{2} \mathrm{~s}$.

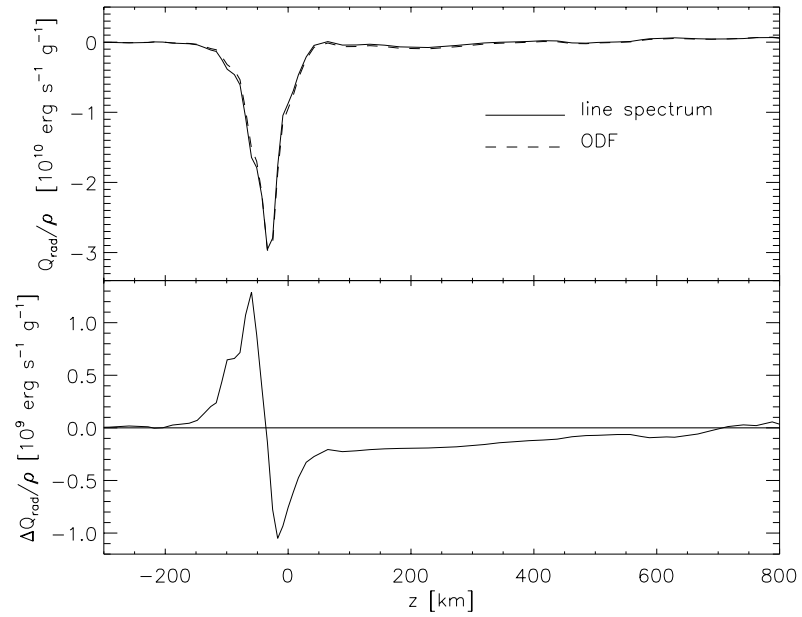

Fig. 19. Upper panel: radiative heating rate per mass unit, $Q_{\mathrm{rad}} / \varrho$, as a function of heigth. The solid line shows the result of a calculation where the detailed opacity spectrum was resolved, the dashed line shows the ODF-based solution. The difference between the two solutions is displayed in the lower panel.

These results of course can only serve as a rough guideline, since it is difficult to predict their relevance in multidimensional moving atmospheres. Nevertheless, they indicate that the error in $Q_{\text {rad }}$ of a 5-bin model should have much less severe consequences for the atmospheric dynamics than that of a grey approximation, making a multigroup approach to the radiative transfer problem appear very favorable.

\subsection{Errors resulting from the ODF approximation}

In the previous sections, ODF-based opacities were used both as the basis for the multigroup sorting process and for calculating the reference radiative heating rates against which the multigroup results were compared. As already mentioned in Sect. 2.1, the use of ODFs incurs an error since the reordering of frequencies within ODF-intervals implicitly relies on the assumption that frequency- and height-dependence of the opacity are decoupled. Figure 19 shows a comparison between the ODF-based $Q_{\text {rad }}$ and the exact solution resulting from a calculation which resolved the detailed frequency-dependence of the line-opacities. The difference between the two solutions is, on the whole, very small. The relatively large errors of the ODF solution around and below $z=0$ mainly result from a small relative shift of the order of $10 \mathrm{~km}$ of the main cooling region around $z=0$. For a large part of the atmosphere above $z=0$, the ODF approximation results in too low values of $Q_{\mathrm{rad}}$, the error decreasing with increasing height. This error is, however, small compared with the errors in the upper atmosphere incurred by multigroup models with 4-6 opacity bins, confirming that the use of ODFs as the reference opacities is justified.

\subsection{Effects of velocity gradients}

Both the ODF approximation and the multigroup method make the implicit assumption that frequency- and height-dependence of the opacity are decoupled within a given ODF-interval or 


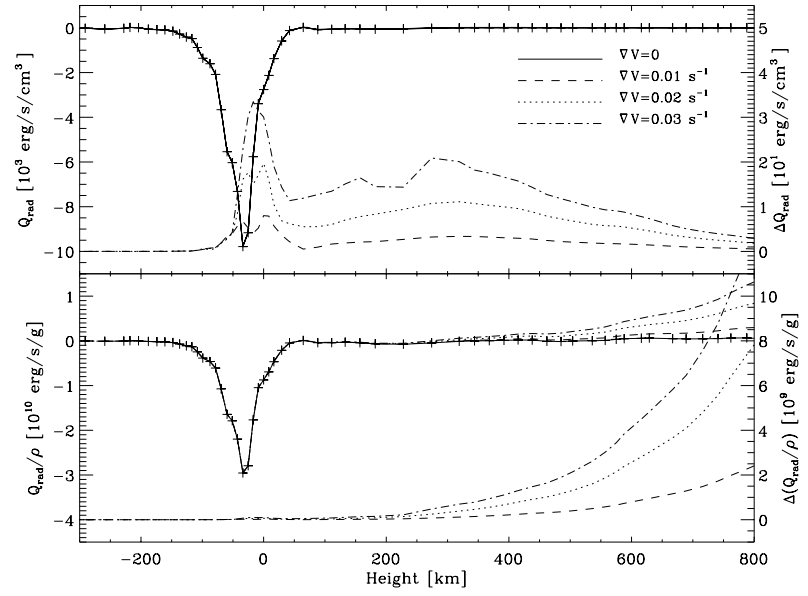

Fig. 20. Radiative heating rates (upper panel: per volume, lower panel: per mass unit) in a 1D atmosphere with a constant (heightindependent) vertical velocity gradient. In both panels, the lower curves show the deviation from the non-moving case.

opacity bin. Therefore neither of these concepts can capture the effects of relative doppler shifts due to velocity gradients upon the radiative heating rate. In order to obtain an estimate of these effects, we imposed a constant (height-independent) vertical velocity gradient on the $1 \mathrm{D}$ solar model atmosphere used in the previous sections. Figure 20 shows the result of calculations based on the detailed spectrum of line-opacities with the doppler shifts taken into account, for different values of the velocity gradient. Over the whole height range above $z=0$ the velocity gradients introduce an additional heating, which becomes increasingly important in the upper parts of the atmosphere as the effect on the radiative heating rate per mass unit increases strongly with height. This heating can be understood as a consequence of "hot" continuum radiation moving in frequency space and getting absorbed by adjacent lines as it travels upwards in the differentially moving atmosphere. Based on characteristic velocities of the order of $1 \mathrm{~km} \mathrm{~s}^{-1}$ and scale heights of $100 \mathrm{~km}$ in the upper photosphere, we estimate typical velocity gradients to be of the order of $0.01 \mathrm{~s}^{-1}$. For gradients of this magnitude, the heating due to doppler shifts is negligible below $z \approx 500 \mathrm{~km}$. In shocks with possibly much steeper gradients, the effect can, however, be expected to be significant. At greater heights it becomes more difficult to assess the importance of gradients based on our LTE calculations, since line-scattering effects, which become important in the chromosphere, tend to reduce the heating effect resulting from true absorption.

\section{Summary and conclusions}

We have performed a series of test calculations which showed that, using the multigroup method, a good approximation to the frequency integrated radiative heating rate can be obtained at moderate computational costs, which makes the multigroup approach the method of choice in multidimensional timedependent MHD simulations.

The advantage over the grey approximation is particularly pronounced in situations which deviate strongly from the one-dimensional plane-parallel case. In these cases the radiative transfer is complicated by lateral heating and cooling effects. We found that the grey radiative transfer fails to capture these effects, which leads to qualitatively wrong heating rates in the upper photosphere. Our results suggest that a grey treatment of the radiative transfer in numerical simulations is insufficient if the simulations extend to the upper photosphere. This is particularly true if magnetic field concentrations are included, since the partial evacuation of these fields leads exactly to the kind of lateral inhomogeneities, which are not well modelled by the grey approach.

Test calculations with snapshots from numerical simulations have shown that the good performance of the multigroup method with $\tau$-sorting does not depend crucially on the choice of the reference atmosphere, which underlines the applicability of this method in realistic multi-dimensional simulations.

We studied the convergence of different multigroup models in the limit of a large number of opacity bins. We present a new approach for defining bin-averaged opacities, which is based on the radiation field in a reference atmosphere, and which can further improve the performance of the multigroup method, particularly if ten or more opacity bins are considered.

Finally, one-dimensional test calculations showed that velocity gradients in the photosphere introduce an additional heating effect which can, however, be neglected in simulations which do not extend beyond a height of approximately $500 \mathrm{~km}$ above continuum optical depth unity at $500 \mathrm{~nm}$.

Acknowledgements. This work has been supported by the Deutsche Forschungsgemeinschaft under grant SCHU 500/7 in the framework of the Priority Research Program "Analysis and Numerics of Conservation Laws". A.V. gratefully acknowledges support by the Max-Planck-Institute for Aeronomy through a doctoral stipend.

\section{References}

Bruls, J. H. M. J., Vollmöller, P., \& Schüssler, M. 1999, A\&A, 348, 233

Carlson, B. G. 1963, in Methods in Computational Physics, ed. B. Alder, \& S. Fernbach, 1

Gustafsson, B., Bell, R. A., Eriksson, K., \& Nordlund, A. 1975, A\&A, 42, 407

Kunasz, P. B., \& Auer, L. 1988, J. Quant. Spectrosc. Radiat. Transfer, 39,67

Kurucz, R. 1993, ATLAS9 Stellar Atmosphere Programs and $2 \mathrm{~km} \mathrm{~s}^{-1}$ grid., Kurucz CD-ROM No. 13, Cambridge, Mass.: Smiths onian Astrophysical Observatory

Lowan, A. N., Davids, N., \& Levenson, A. 1942, Bull. Amer. Math. Soc., 48,739

Ludwig, H. G. 1992, Ph.D. Thesis

Ludwig, H.-G., Jordan, S., \& Steffen, M. 1994, A\&A, 284, 105

Maltby, P., Avrett, E. H., Carlsson, M., et al. 1986, ApJ, 306, 284

Mihalas, D. 1967, ApJ, 149, 169

Mihalas, D. 1978, Stellar atmospheres, 2nd edition (San Francisco: W. H. Freeman and Co)

Nordlund, A. 1982, A\&A, 107, 1

Peytremann, E. 1974, A\&A, 33, 203

Ploner, S. R. O., Solanki, S. K., \& Gadun, A. S. 1999, A\&A, 352, 679

Skartlien, R. 2000, ApJ, 536, 465

Sneden, C., Johnson, H. R., \& Krupp, B. M. 1976, ApJ, 204, 281

Strom, S. E., \& Kurucz, R. 1966, AJ, 71, 181 